\title{
1 Transcriptome and histone epigenome of Plasmodium vivax 2 salivary-gland sporozoites point to tight regulatory control 3 and potential mechanisms for liver-stage differentiation.
}

4

5

\author{
Vivax Sporozoite Consortium* (Ivo Muller ${ }^{1,2,3}$, Aaron R. Jex ${ }^{1,3,4}$, Stefan H. I. Kappe ${ }^{5}$, \\ Sebastian A. Mikolajczak ${ }^{5}$, Jetsumon Sattabongkot ${ }^{7}$, Rapatbhorn Patrapuvich ${ }^{6}$, Scott \\ Lindner $^{8}$, Erika L. Flannery ${ }^{5}$, Cristian Koepfli ${ }^{1}$, Brendan Ansell ${ }^{4}$, Anita Lerch ${ }^{1}$, Samantha J \\ Emery-Corbin ${ }^{1}$, Sarah Charnaud ${ }^{1}$, Jeffrey Smith $^{1}$, Nicolas Merrienne ${ }^{2}$, Kristian E. \\ Swearingen ${ }^{5}$, Robert L. Moritz ${ }^{9}$, Michaela Petter ${ }^{10,11}$, Michael Duffy ${ }^{10}$, Vorada Chuenchob ${ }^{5}$ ). \\ "Group authorship - all authors are equal contributors (order per author contributions section below). \\ 1. Population Health and Immunity Division, The Walter and Eliza Hall Institute for Medical Research, 1G Royal Parade, \\ Parkville, Victoria, 3052, Australia. \\ 2. Malaria: Parasites \& Hosts Unit, Institut Pasteur, 28 Rue de Dr. Roux, 75015, Paris, France. \\ 3. Department of Medical Biology, The University of Melbourne, Victoria, 3010, Australia. \\ 4. Faculty of Veterinary and Agricultural Sciences, The University of Melbourne, Corner of Park and Flemington Road, \\ Parkville, Victoria, 3010, Australia. \\ 5. Center for Infectious Disease Research, 307 Westlake Avenue North, Suite 500, Seattle, WA 98109, USA; \\ 6. Department of Global Health, University of Washington, Seattle, WA 98195, USA. \\ 7. Mahidol Vivax Research Center, Faculty of Tropical Medicine, Mahidol University, Bangkok 10400, Thailand. \\ 8. Department of Biochemistry and Molecular Biology, Center for Malaria Research, Pennsylvania State University, University \\ Park, PA 16802, USA. \\ 9. Institute for Systems Biology, Seattle, WA, 98109, USA. \\ 10. Department of Medicine Royal Melbourne Hospital, The Peter Doherty Institute, The University of Melbourne, 792 \\ Elizabeth Street, Melbourne, Victoria 3000, Australia. \\ 11. Institute of Microbiology, University Hospital Erlangen, Erlangen 91054, Germany
}

\begin{abstract}
Plasmodium vivax is the key obstacle to malaria elimination in Asia and Latin America, largely attributed to its ability to form resilient hypnozoites (sleeper-cells) in the host liver that escape treatment and cause relapsing infections. The decision to form hypnozoites is made early in the liver infection and may already be set in sporozoites prior to invasion. To better understand these early stages of infection, we undertook a comprehensive transcriptomic and histone epigenetic characterization of $P$. vivax sporozoites. The salivarygland sporozoite transcriptome is heavily composed of transcripts associated with functions needed for early infection of the vertebrate host and development within hepatocytes. Through comparisons to recently published proteome data for the $P$. vivax sporozoite, our study finds that although highly transcribed, these transcripts are not detectable as proteins and may be regulated through translational repression; a finding we test for a small subset of transcripts and proteins through immunofluorescent microscopy of sporozoites and liver stages in humanized mice. We identify differential transcription between the sporozoite and published transcriptomes of asexual blood-stages and mixed versus hypnozoite-enriched liver stages. These comparisons point to multiple layers of transcriptional, post-transcriptional and post-translational control that appear active in sporozoites and to a lesser extent hypnozoites, but largely absent in replicating liver schizonts or mixed blood-stages. Common transcripts up-regulated in sporozoites and hypnozoites compared to mixed (i.e., schizont) liver-stages identify genes linked to dormancy/persistence in bacteria, amoebae and plants. We also characterise histone epigenetic modifications in the $P$. vivax sporozoite and explore their role in regulating transcription. Collectively, these data support the hypothesis that the sporozoite as a tightly programmed stage primed to infect the human host and identifies potential mechanisms for hypnozoite-formation that may be further explored in liver stage models.
\end{abstract}

\section{INTRODUCTION}

Malaria is among the most significant infectious diseases impacting humans globally, with 3.3 billion people at risk of infection, 381 million suspected clinical cases and up to $~ 660,000$ deaths attributed to malaria in 2014 [1]. Two major parasitic species contribute to the vast majority of human malaria, Plasmodium falciparum and $P$. vivax. Historically, P. falciparum has attracted the majority of global attention, due to its higher contribution to morbidity and 
mortality. However, $P$. vivax is broadly distributed, more pathogenic than previously thought, and is recognised as the key obstacle to malaria elimination in the Asia-Pacific and Americas [2]. Unlike $P$. falciparum, $P$. vivax can establish long-lasting 'sleeper-cells' (= hypnozoites) in the host liver that emerge weeks, months or years after the primary infection (= relapsing malaria) [3]. Primaquine is the only approved drug that prevents relapse. However, the short half-life, long dosage regimens and incompatibility of primaquine with glucose-6-phosphatedehydrogenase deficiency (which requires pre-screening of recipients [4]) makes it unsuitable for widespread use. As a consequence, $P$. vivax is overtaking $P$. falciparum as the primary cause of malaria in a number of co-endemic regions [5]. Developing new tools to diagnose, treat and/or prevent hypnozoite infections is considered one of the highest priorities in the malaria elimination research agenda [6].

When Plasmodium sporozoites are deposited by an infected mosquito, they likely traverse the skin cells, enter the blood-stream and are trafficked to the host liver, as has been shown in rodents [7]. The sporozoites' journey from skin deposition to hepatocytes takes less than a few minutes [8]. Upon reaching the liver, sporozoites traverse Kupffer and endothelial cells to reach the parenchyma, moving through several hepatocytes before invading a final hepatocyte suitable for development [7,9]. Within hepatocytes, these parasites replicate, and undergo further development and differentiation to produce merozoites that emerge from the liver and infect red blood cells. However, $P$. vivax sporozoites are able to commit to two distinct developmental fates within the hepatocyte: they either immediately continue development as replicating schizonts and establish a blood infection, or delay replication and persist as hypnozoites. Regulation of this major developmental fate decision is not understood and this represents a key gap in current knowledge of $P$. vivax biology and control.

Sporozoites prepare for mammalian host infection while still residing in the mosquito salivary glands. It has been hypothesized that $P$. vivax sporozoites exist within an inoculum as replicating 'tachysporozoites' and relapsing 'bradysporozoites' [10] and that these subpopulations may have distinct developmental fates as schizonts or hypnozoites, thus contributing to their relapse phenotype [10-12]. This observation is supported by the stability of different hypnozoite phenotypes (ratios of hypnozoite to schizont formation) in $P$. vivax infections of liver-chimeric mouse models [13]. To determine fates in the sporozoite stage control of protein expression must take place. Studies using rodent malaria parasites have identified genes [14] that are transcribed in sporozoites but translationally repressed (i.e., present as transcript but un- or under-represented as protein), via RNA-binding proteins [15], and ready for immediate translation after the parasites' infection of the mammalian host cell $[13,16]$. It is therefore also possible that translational repression (i.e., the blocking of translation of present and retained transcripts) and other mechanisms of epigenetic control may contribute to the $P$. vivax sporozoite fate decision and hypnozoite formation, persistence and activation. Supporting this hypothesis, histone methyltransferase inhibitors stimulate increased activation of $P$. cynomolgi hypnozoites to become schizonts in macaque hepatocytes [17, 18]. Epigenetic control of stage development is further evidenced in Plasmodium through chromatin structure controlling expression of PfAP2-G, a specific transcription factor that, in turn, regulates gametocyte (dimorphic sexual stages) development in blood-stages [19]. It is well documented that $P$. vivax hypnozoite activation patterns stratify with climate and geography [11] and recent modelling suggests transmission potential selects for hypnozoite phenotype [20]. Clearly the ability for $P$. vivax to dynamically regulate hypnozoite formation and relapse phenotypes in response to high or low transmission periods in different climate conditions would confer a significant evolutionary advantage.

Unfortunately, despite recent advances [21] current approaches for in vitro P. vivax culture do not support routine maintenance in the laboratory and tools to directly perturb gene function are not established. This renders studies on $P$. vivax, particularly its sporozoites and liver stages, exceedingly difficult. Although in-vitro liver stage assays and humanised mouse models are being developed [13], 'omics analysis of $P$. vivax liver stage dormancy has until recently [22] been impossible and even now is in its early stages. Recent characterization [23] of liver-stage (hypnozoites and schizonts) of $P$. cynomolgi (a related and relapsing parasite in macaques) provides valuable insight, but investigations in $P$. vivax directly are clearly 
114 needed. The systems analysis of $P$. vivax sporozoites that reside in the mosquito salivary glands and are poised for transmission and liver infection offer a key opportunity to gain insight into $P$. vivax infection. Plasmodium vivax sporozoites have been explored previously by microarray [24] and most recently, in a single RNA-seq replicate [25] and a study on sporozoite activation $\{$ Roth, $2018 \# 66\}$. Epigenetic regulation in sporozoites has only been explored in $P$. falciparum [26, 27]. Here, we present a detailed characterization of the $P$. vivax sporozoite transcriptome and histone epigenome and use these data to better understand this key infective stage and the role of sporozoite programming in invasion and infection of the human host, and development within the host liver.

\section{RESULTS AND DISCUSSION}

Mosquito infections were generated by membrane feeding of blood samples taken from $P$. vivax infected patients in western Thailand $(\mathrm{n}=9)$. Approximately 3-15 million $P$. vivax sporozoites were harvested per isolate from Anopheles dirus salivary glands. Using RNA-seq, we detected transcription for 5,714 P. vivax genes (based on the P. vivax $\mathrm{P} 01$ gene models: [28]) and obtained a high degree of coverage $(4,930$ with a mean counts per million (CPM) $\geq$ 1.0; Figure S1 and Table S1 and S2). Among the most highly transcribed genes in the infectious sporozoite stage are $c s p$ (circumsporozoite protein), five etramps (early transcribed membrane proteins), including uis3 (ㅁp-regulated in infective sporozoites), uis4 and lsap-1 (liver stage associated protein 1), a variety of genes involved in cell transversal and initiation of invasion, including celtos (cell traversal protein for ookinetes and sporozoites), gest (gamete egress and sporozoite traversal protein), spect1 (sporozoite protein essential for cell traversal) and siap-1 (sporozoite invasion associated protein), and genes associated with translational repression (albal, alba4 and Puf2). Collectively, these genes account for $>1 / 3^{\text {rd }}$ of all transcripts in the sporozoite. Although we found only moderate agreement $\left(\mathrm{R}^{2}=0.35\right.$; Figure S2) between our RNA-seq data and previous microarray data for $P$. vivax sporozoites and blood-stages [24], improved transcript detection and quantitation is expected with the increased technical resolution of RNA-seq over microarray. Supporting this, we find higher correlation between RNA-seq data from $P$. vivax and $P$. falciparum (single replicate sequenced herein for comparative purposes) sporozoite datasets $\left(\mathrm{R}^{2}=0.42\right)$, compared to either species relative to published microarray data (Figure S2 and Table S3).

Although microarray supports the high transcription in sporozoites of genes such as uis 4 , csp, celtos and several other etramps, $27 \%$ and $16 \%$ of the most abundant $1 \%$ of transcribed genes in our sporozoite RNA-seq data are absent from the top decile or quartile respectively in the existing $P$. vivax sporozoite microarray data [24]. Among these are genes involved in early invasion/hepatocyte development, such as lsap-1, celtos, gest and siap-1, or translational repression (e.g., alba-1 and alba-4); orthologs of these genes are also in the top percentile of transcripts in RNA-seq (see [26, 29]) and previous microarray data [30, 31] for human-infecting $P$. falciparum and murine-infecting $P$. yoelii sporozoites, suggesting many are indeed more abundant than previously characterized. A subset of representative transcripts, including Pv_AP2-X (PVP01_0733100), d13, gest, g10 (PVP01_1011100), 40S ribosomal protein S27 (PVP01_1409300), puf-2, zipco and 14-3-3, were tested by qPCR for their transcript abundance relative to celtos and sera (Figure 1A and Table S4). This representative set differed markedly in their relative abundance between our RNAseq and previous microarray data [24]. To control for batch effects introduced by collection of the sporozoites used here for RNAseq, this testing was conducted in an additional six sample replicates representing four additional clinical P. vivax isolates (PvSPZ-Thai13-16; with PvSPZ-Thai16 tested in technical triplicate). The qPCR results agreed with the RNAseq data for these transcripts (Table S4).

Transcription in P. vivax relative to other plasmodia sporozoites. To gain insight into species-specific aspects of the P.vivax transcriptome, we qualitatively compared these data with available data for $P$. falciparum [27] and $P$. yoelii sporozoites (single replicate only) for 4,067 single-copy orthologs (SCO) (transcribed at $\geq 1$ TPM in $P$. vivax infectious sporozoites) shared with $P$. falciparum and $P$. yoelli (Table S5). Genes highly transcribed in 
169 salivary-gland sporozoites of all three species include celtos, gest, trap, siap1, spect1 and puf2. There are 696 P. vivax genes shared as orthologs between $P$. vivax $\mathrm{P} 01$ and $P$. vivax Sall lacking a defined SCO in P. falciparum or $P$. yoelli transcribed at a mean of $\geq 1$ TPM in $P$. vivax salivary-gland sporozoites (Table S6). Prominent among these are $\operatorname{vir}(\mathrm{n}=25)$ and $P v$ fam (41 fam-e, 16 fam-b, 14 fam-a, 8 fam-d and 3 fam-h) genes, as well as hypothetical proteins or proteins of unknown function $(\mathrm{n}=212)$ and, interestingly, a number of 'merozoite surface protein' 3 and 7 homologs ( $\mathrm{n}=5$ of each). Both $m s p 3$ and $m s p 7$ have undergone significant expansion in $P$. vivax relative to $P$. falciparum and $P$. yoelii [32] and may have repurposed functions in sporozoites. In addition, there are $69 P$. vivax $\mathrm{P} 01$ genes lacking a defined ortholog in $P$. vivax Sall, $P$. falciparum or $P$. yoelli transcribed at $\geq 1$ TPM in infectious $P$. vivax sporozoites; most of which are Plasmodium interspersed repeat (PIR) genes [32] found in telomeric regions of the P01 assembly and likely absent from the Sal1 assembly but present in the Sall genome, indicating the improved coverage of telomeric regions in P01 relative to Sal1.

P. vivax sporozoites transcriptome compared with proteome. We compared relative protein abundance presented in a recently published $P$. vivax sporozoite proteome [33] to relative transcript abundance from the current study (Figure 1B and Table S7). The proteome study incorporated data from the same PvSPZ-Thail and PvSPZ-Thai5 isolates tested by RNAseq here. We identified 2,402 P. vivax genes transcribed in the sporozoite $(\mathrm{CPM}>1)$ for which no protein expression was detected. Although many of these are lowly transcribed and likely below the detection sensitivity of LC-MS proteomics, others are among the most highly transcribed genes in the sporozoite, indicating these may be under translational repression.

Translational repression, the mechanism through which transcripts are held in stasis by RNA binding proteins, has been demonstrated to have important functional roles in the transition of Plasmodium spp. between the vertebrate to invertebrate host. More than 700 genes have been identified as translationally repressed in Plasmodium berghei ('rodent malaria') gametocytes based on DOZI (DEAD box RNA helicase "development of zygote inhibited") pulldowns [34]. Translational repression mechanisms mediated through Puf-2 have been explored in sporozoites of several Plasmodium species and regulate some of the most abundant transcripts in the sporozoite, such as uis-3 and uis-4. UIS3 and UIS4 are the best characterized proteins under translational repression by Puf-2 in sporozoites [35] and are essential for liver-stage development [14].

In considering genes that may be translationally repressed (i.e., transcribed but not translated) in the $P$. vivax sporozoite, we confine our observations to those transcripts representing the top decile of transcript abundance to ensure their lack of detection as proteins was not due to limitations in the detection sensitivity of the proteomic dataset. Approximately $1 / 3^{\text {rd }}$ of transcripts in the top decile of transcriptional abundance $(n=170$ of 558) in P. vivax sporozoites were not detectable as peptides in multiple replicates (Figure 1B and Table S7). Of these 170 putatively repressed transcripts, 156 and 154 have orthologs in P. falciparum and $P$. yoelii respectively, with 89 and 118 of these also not detected as proteins in $P$. falciparum and $P$. yoelii salivary-gland sporozoites [36] despite being highly transcribed in these stages (see [26, 29]; Tables S3-S5), and 133 (78.2\%) having no detectable sporozoite expression ( $>1$ unique peptide count) in LC-MS data deposited for any species in PlasmoDB (Table S8). In contrast, 106 of these putatively repressed transcripts with orthologs in other Plasmodium species (Table S8) for which proteomic data is available in PlasmoDB, are detectable ( $>1$ unique peptide count) by LC-MS methods in at least one other life-cycle stage, indicating against a technical issue (e.g., inability to be trypsin-digested) preventing their detection in the P. vivax sporozoite proteome [33]. In addition to uis3 and uis4, genes involved in liver stage development and detectable as transcripts but not proteins in the $P$. vivax sporozoites include lsapl (liver stage associated protein 1), zipco (ZIP domaincontaining protein), several other etramps (PVP01_1271000, PVP01_0422600, PVP01_0504800 and PVP01_0734800), pv1 (parasitophorous vacuole protein 1) and lisp1 and lisp2 (PVP01_1330800 and PVP01_0304700). Also notable among genes detectable as transcripts but not proteins in sporozoites is a putative 'Yippee' homolog (PVP01_0724100). 
224 Yippee is a DNA-binding protein that, in humans (YPEL3), suppresses cell growth [37] and

225

226

227

228

229

230

231

232

233

234

235

236

237

238

239

240

241

242

243

244

245

246

247

248

249

250

251

252

253

254

255

256

257

258

259

260

261

262

263

264

265

266

267

268

269

270

271

272

273

274

275

276

277

278 is regulated through histone acetylation [38], making it noteworthy in the context of $P$. vivax hypnozoite developmental arrest.

Although verifying each putatively repressed transcript will require further empirical data, our system level approach is supported by immunofluorescent microscopy (Figure 1C) of UIS4, LISP1, EXP1 and ACP (PVP01_0416300). These represent one known and three putative (i.e., newly proposed here) translationally repressed genes in $P$. vivax sporozoites, and are compared to TRAP and $\mathrm{BiP}$ (which are both transcribed and expressed as protein in the $P$. vivax sporozoite; Table S8). The lispl gene is an interesting find. In P. berghei, lispl is essential for rupture of the PVM during liver stage development allowing release of the merozoite into the host blood stream. Pv-lispl is $\sim 350$-fold and $\sim 1,350$-fold more highly transcribed in $P$. vivax sporozoites compared to sporozoites of either $P$. falciparum or $P$. yoelii (see Table S5). IFAs using LISP1 specific mAbs (Figure 1C) show that this protein is undetectable in sporozoites but clearly expressed at 7 days post-infection in liver schizonts.

Up-regulated transcripts in $P$. vivax sporozoites relative to other life-cycle stages. Recently completed studies of the transcriptome of $P$. vivax for sporozoite activation [39], as well as, liver [22] and asexual blood-stages [40] support comparative transcriptomic study of sporozoites, their biology and transcriptional regulation over the $P$. vivax life-cycle. Recently published data for activate sporozoites from Roth et al [39] was significantly lower depth coverage, with $\sim 0.03$ to $0.6 \mathrm{M}$ reads mapped the $P$. vivax $\mathrm{P} 01$ coding domains; compared with 0.7 to $15.3 \mathrm{M}, 2.4$ to $10.6 \mathrm{M}$ and 18.7 to $57.6 \mathrm{M}$ mapped reads for salivary sporozoite, liverstages [22] and asexual blood stages [40] respectively. This lower coverage could not be compensated for through data normalization and therefore data from Roth et al [39] was not included in our quantitative analyses, although qualitatively, many of the highly transcribed genes in Roth et al [39] sporozoites were among the highly transcribed genes in salivary sporozoites from the present study. The remaining RNAseq data presents an analytical challenge in that each (sporozoites, liver-stages and blood stages) is produced in a separate study and may be influenced by technical batch effects that cannot be differentiated from biologically meaningful changes. To address this, we first examined $P$. vivax transcripts in a previous microarray study of multiple $P$. vivax life-cycle stages [24], including sporozoites and several blood-stages, to identify genes that may be transcriptionally stable across the lifecycle. We identified $\sim 160$ genes with low transcriptional variability between sporozoites and blood-stages that covered the breadth of transcript abundance levels in Westenberger et al [24]. These include genes typically associated with "house-keeping" functions, such as ribosomal proteins, histones, translation initiation complex proteins and various chaperones (see Figure S3 and Table S8). We assessed transcription of these 160 genes among the current and recently published RNA-seq data for $P$. vivax and all were of similarly low variability (Figure S4). This suggests that any batch effect between the studies is sufficiently lower than the biological differences between each life-cycle stage, allowing informative comparisons. We then combined all published RNAseq-based, transcriptomic data available for $P$. vivax $[22,39,40]$ with the salivary sporozoite data generated here.

P. vivax sporozoite relative to blood-stage transcriptome. To identify transcripts upregulated in sporozoites, we first compared the $P$. vivax sporozoite transcriptome to RNA-seq data for P. vivax blood-stages [40] (Figure 2 and Figures S7-S10). We identified 1,672 up (Table S9); Interactive Glimma Plot - Supplementary Data 1) and 1,958 down-regulated (Table S9); Interactive Glimma Plot - Supplementary Data 1) transcripts (FDR $\leq 0.05$; minimum 2-fold change in Counts per Million (CPM)) and explored patterns among these differentially transcribed genes (DTGs) by protein family (Figure 2C and Table S10) and Gene Ontology (GO) classifications (Table S11). RNA recognition motifs (RRM-1 and RRM-6) and helicase domains (Helicase-C and DEAD box helicases) are over-represented (p-value <0.05) among transcripts up-regulated in sporozoites, consistent with translational repression through ribonucleoprotein (RNP) granules [41]. Transcripts encoding nucleic acid binding domains, such as bromodomains (PF00439; which can also bind lysine-acetylated 
proteins), zinc fingers (PF13923) and EF hand domains (PF13499) are also enriched in sporozoites. Included among these proteins are a putative ApiAP2 transcription factor (PVP01_1211900) and a homologue of the Drosophila zinc-binding protein 'Yippee' (PVP01_0724100). Thrombospondin-1 like repeats (TSR: PF00090) and von Willebrand factor type A domains (PF00092) are enriched in sporozoites as well. In P. falciparum sporozoites, genes enriched in TSR domains are important in invasion of the mosquito salivary gland (e.g., trap) and secretory vesicles released by sporozoites upon entering the vertebrate host (e.g., csp) [42]. By comparison, genes up-regulated in blood-stages are enriched for vir gene domains (PF09687 and PF05796), Tryptophan-Threonine-rich Plasmodium antigens (PF12319; which are associated with merozoites [43]), markers of celldivision (PF02493; [44]) protein production/degradation (PF00112, PF10584, PF00152, PF09688 and PF00227) and ATP metabolism (PF08238 and PF12774). 47 of the 343 transcripts unique to $P$. vivax sporozoites relative to $P$. falciparum or $P$. yoelii are upregulated in sporozoites compared to $P$. vivax blood stages. Nine of these are in the top decile of transcription, and include a Pv-fam-e (PVP01_0525200), a Pf-fam-b homolog (PVP01_0602000) and 7 proteins of unknown function. A further nine have an ortholog in $P$. cynomolgi (which also forms hypnozoites) but not the closely related $P$. knowlesi (which does not form hypnozoites) and include 'msp7'-like (PVP01_1219600, PVP01_1220300 and PVP01_1219900), 'msp3'-like (PVP01_1031300), Pv-fam-e genes (PVP01_0302100, PVP01_0524500 and PVP01_0523400), a serine-threonine protein kinase (PVP01_0207300) and a RecQ1 helicase homolog (PVP01_0717000). Notably, the P. cynomolgi ortholog of PVP01_0207300, PCYB_021650, is transcriptionally up-regulated in hypnozoites relative to replicating schizonts [23], indicating a target of significant interest when considering hypnozoite formation and/or biology and suggesting that the list here may contain other genes important in hypnozoite biology.

P. vivax sporozoites are enriched in translational repressors. In Plasmodium, translational repression regulates key life-cycle transitions coinciding with switching between the mosquito and the mammalian host (either as sporozoites or gametocytes) [41]. For example, although uis 4 is the most abundant transcript in the infectious sporozoite $([24,31]$; Table S2), UIS4 is translationally repressed in this stage [15] and only expressed after hepatocyte invasion [45]. In sporozoites, it is thought that PUF2 binds to mRNA transcripts and prevents their translation [29], and SAP1 stabilises the repressed transcripts and prevents their degradation [45]. Consistent with this, Puf2 and SAP1 (PVP01_0947600) are up-regulated in the sporozoite relative to blood-stages. Indeed, Puf2 (PVP01_0526500) is among the top percentile of transcripts in salivary sporozoites and expressed at high levels in the proteome [33]. However, our data implicate other genes that may act in translational repression in $P$. vivax sporozoites, many of which are already known to be involved in translational repression in other Plasmodium stages and other protists [41]. Among these are alba-2 and alba-4, both of which are among the top $2 \%$ of genes transcribed in sporozoites and $~ 14$ to 20 -fold more highly transcribed in sporozoites relative to blood-stages; ALBA-2 is in the top 100 most abundant proteins in the P. vivax sporozoite proteome [33]. In addition, P. vivax sporozoites are enriched for genes encoding RRM-6 RNA helicase domains. Intriguing among these are $\mathrm{HoMu}$ (homolog of Musashi; top decile of sporozoite proteins by abundance [33]) and ptbp

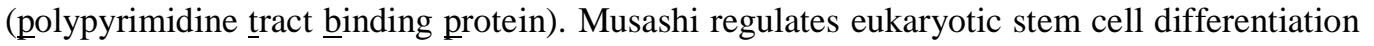
through translational repression [46] and HoMu localizes with DOZI and CITH in Plasmodium gametocytes [47]. PTBP is linked to mRNA stability, splice regulation and translational initiation [48] and may perform a complementary role to SAP1.

P. vivax sporozoite relative to Plasmodium spp. liver stage transcriptomes. New advances in $P$. vivax liver culture has allowed recent publication of mixed stage and hypnozoite-enriched transcriptomes [22]. This is an early, yet highly valuable, study and, due no doubt to the difficulty in generating the material, is limited to biological duplicates. Noting this, although we undertake differential transcriptomic studies of this dataset here, we recognize that additional biological replication is needed and have used a higher burden of significance 
334 (FDR $\leq 0.01$ and $\geq 2$-fold change) than used with blood-stages. Nevertheless, these

335

336

337

338

339

340

341

342

343

344

345

346

347

348

349

350

351

352

353

354

355

356

357

358

359

360

361

362

363

364

365

366

367

368

369

370

371

372

373

374

375

376

377

378

379

380

381

382

383

384

385

386

387

388 comparisons identified 1,015 and 856 sporozoite up-regulated transcripts relative to mLS and HPZs respectively and 1,007 and 1,079 transcripts up-regulated in mLS and HPZs relative to sporozoites respectively (Figures 3 and S11-S13, Table S12 and S13 and Interactive Glimma Plot - Supplementary Data 1).

Compared to mLS transcriptomes, sporozoites are enriched for many of the transcripts similarly up-regulated in comparison to blood-stages (e.g., uis4, celtos, puf2, siap1 and $p l p-1)$. More broadly, SPZ up-regulated transcripts over-represent (p-value $\leq 0.05$ ) Pfam domains (Figure 3A) associated with transcriptional regulation (PF00176, PF01096, PF01661 and PF08711), translational repression/regulation (PF00076, PF00279, PF01008, PF01873, PF01917, PF02847, PF02854, PF13893 and PF14259), DNA/RNA binding (PF0097, PF13445, PF13639, PF14570 and PF15247) and chromatin regulation (PF00271, PF00850 and PF13489). In contrast, the mLS transcriptome is enriched in genes involved in replication and merozoite formation [ $\mathrm{n}=14$; including PVP01_0728900 (mspl), PVP01_0010670 (msp3) and PVP01_1446800 (msp9)], rhoptry function [ $\mathrm{n}=9$; including PVP01_1469200 (rnp3), PVP01_1255000 (rnp2) and PVP01_1338500 (rapl)] and reticulocyte binding [n=10 including PVP01_0534300 (rbp2c), PVP01_1402400 (rbp2a), PVP01_0701100 (rbp1b) and PVP01_0800700 (rbp2b)]. These data are further enriched for Pfam domains associated with cell division (PF00493), merozoite formation (PF07133 and PF12984), proteasome function (PF00227, PF00400, PF00656, PF01344, PF01398 and PF03981), protein export / vesicle function (PF00350 and PF00996), membrane proteins (PF01105, PF03011, PF05424 and PF12139) and metabolism (PF00085, PF00118, PF00268, PF01066, PF01214 and PF01214). Collectively, in addition to markers consistent with sporozoite or merozoite formation, these data point towards the sporozoite stage as being highly regulated and controlled at transcriptional, translational and chromatin levels, with the mLS stages representing a release of this control allowing replication, protein turn-over, reconfiguration of the proteins on the plasma membrane and metabolic activity.

Comparison of sporozoites with HPZs does not indicate a similar release of control, or at least that any release is more specific than for mLS. The sporozoite is enriched, relative to HPZs, in genes such as PVP01_1258000 (gest), PVP01_0418000 (sera), PVP01_1435400 (celtos), PVP01_0835600 (csp) and PVP01_0602100 (uis4). At a broad level, sporozoite enriched Pfam domains include a smaller number associated with translational repression/regulation (PF00076) or DNA/RNA binding (PF01428 and PF12756). Interestingly, sporozoites are enriched in Pfam domains specifically associated with heterochromatin (H3K9me3) reading/interaction (PF02463, PF00628, PF13831 and PF13865). Ours (see below) and previous epigenetic studies of Plasmodium sporozoites [27] find dense heterochromatin in the telomeric to subtelomeric regions of the chromosome, which is more transcriptionally active in blood-stages [49]. Others have noted an upregulation of methyl/acetyltransferases in $P$. cynomolgi HPZs [23] and/or shown methyltransferase inhibitors stimulate hypnozoite activation in vitro [17]. The potential that histone epigenetics of sporozoites has a role in or changes with liver-stage development and the formation of liver schizonts or HPZs is intriguing but requires detailed study of the chromatin of liver-stage parasites, which is not presently available for $P$. vivax. In contrast, HPZs were enriched, relative to sporozoites, for genes including histone proteins (PVP01_1138700, PVP01_1131700 and PVP01_0905900) and classic markers of metabolism (PVP01_MITO3300 and PVP01_MITO3400) and lisp2. Pfam data indicated- largely similar domain enrichment trends as were seen for the mLS stage relative to sporozoites, including a number of proteosomal (PF00227, PF00112, PF03981), vesicular transport (PF00996) and metabolic (PF00118, PF00268, PF01066, PF01214 a) associated functions. This supports HPZs being an arrested, rather than classically 'dormant', stage with active metabolism and protein turn-over. HPZs are also enriched for Pfams associated with mRNA/tRNA regulation and turnover (PF04857, PF01612, PF00009 and PF01138) and glycine metabolism (PF01571 and PF00464) and acetyl-CoA production (PF02779 and PF00676).

Finally, although not the focus of this study, we looked at differential transcription between mLS and HPZ stages using the Gural et al [22] data, but using the same approaches 
389 as employed here. In particular, we were interested in what these comparisons might provide 390 in terms of sporozoite differentiation or development into liver schizonts or HPZs (Table 391 S14). Among mLS up-regulated transcripts are genes associated with rhoptry function $(\mathrm{n}=$ 11; including PVP01_0107500, PVP01_1469200 and PVP01_1469200), cytoadherence to red-cells (PVP01_1401400 and PVP01_0734500), merozoite formation (PVP01_0728900 and PVP01_0612400) and exported proteins $(\mathrm{n}=6$; including PVP01_0504000, PVP01_0119200 and PVP01_0801600). Consistent with P. cynomolgi [23], HPZ upregulated transcripts include several key sporozoite transcripts, specifically uis4 (PVP01_0602100), puf1 (PVP01_1015000) and speld (PVP01_0938800). At the Pfam domain level, mLS is enriched for metabolic (PF00317) and proteosomal (PF00112) domains also enriched in $\mathrm{mLS}$ or HPZs relative to sporozoites above, as well as domains associated with merozoite formation (PF12948, PF07462), rhoptry function (PF0712), DNA/RNA binding (PF12756, PF10601 and PF02151) and cell division, development and DNA replication (PF06705, PF00533, PF00488, PF02460, PF07034, PF02181). In contrast, HPZs are enriched in Pfam domains that overlap notably with key sporozoite markers, including etramps (PF09716) and puf proteins (PF00806), as well as domains associated with calcium (PF08683) and nucleotide metabolism (PF06437). These data largely indicate that the hypnozoite bears similarity both to the sporozoite and liver schizonts consistent with a stalled stage on the path to schizont development regulated by checkpoint signals that halt/restart normal schizont development, which has been proposed previously for this species [24].

With this is mind, we looked at transcripts that are differentially transcribed in mLS, but not HPZs, relative to SPZs. There are 107 transcripts down-regulated in mLS relative to SPZs that are transcribed at roughly similar levels in both SPZs and HPZs (Figure 3B). A common theme among many of these genes are their role in transcriptional, posttranscriptional, translational or post-translational regulation. Among transcriptional regulators are transcription factors including AP2-SP2 (PVP01_0303400) and three non-AP2-like transcription factors (PVP01_0306600, PVP01_0204300 and PVP01_1415800). Posttranscriptional controllers include several DNA/RNA-binding proteins (PVP01_1011000. PVP01_0932900, PVP01_0715300, PVP_1242600 and PVP01_0605200), RNA helicases (PVP01_1403600 and PVP01_1329800) and mRNA processing (PVP01_1443100 and PVP01_1458200) genes. Translational control includes several key regulators of translation initiation (PVP01_1467700), tRNA processing (PVP01_0318700 and PVP01_1017700) or ribosomal function/biogenesis (PVP01_1443700, PVP01_0421400, PVP01_1117200 and PVP01_0215100). Post-translational control includes two methyltransferases (PVP01_1428800 and PVP01_1465200), including CARM1, which methylates of H3R17 and, in mice, prevents differentiation in embryonic stem cells [50], and a putative histone methylation reading enzyme, EEML2 (PVP01_1014100). The remaining genes in this group have three noteworthy and largely overlapping themes: (1) an association with calcium binding, metabolism or signalling, (2) a role in organellar metabolism and (3) homologs in other organisms, including a variety of prokaryotes and eukaroytes, with key roles in germination, dormancy and persistent non-replicating stages. The latter most function is clearly intriguing in the context of HPZ formation and activation. These genes include a homolog of dihydrolipoamide acyltransferase (aka 'sucB'), which is essential for growth in Mycobacterium tuberculosis [51] and a key regulator in persistent Escherichia coli stages [52]. Another example is gamete fusion factor HAP2, which, despite the name, has been shown to regulate dormancy in eukaryotes ranging from plants [53, 54] to amoebae [55].

In addition to data for $P$. vivax, two transcriptomic studies are now available for $P$. cynomolgi $[27,56]$ that compare mixed/schizont stage parasites with small-form "hypnozoites". In comparing $P$. cynomolgi liver-stage RNA-seq and $P$. vivax liver-stage microarray data, Cubi et al [23] noted a moderate to good level of agreement $\left(\mathrm{R}^{2}=0.50\right)$ as evidence of $P$. cynomolgi being predictive and representative of $P$. vivax. However, Voorberg van der Wel et al [56] explored congruence between their and the Cubi et al [23] studies and found generally good agreement among schizonts and overall relatively poor agreement among hypnozoites from each study. This highlights the complexity of these datasets and indicates caution in comparing the current data to $P$. vivax. ApiAP2 transcription factors 
444 feature prominently in each liver-stage transcriptomic study for $P$. cynomolgi $[23,56]$ and $P$. 445 vivax [22]. Cubi et al [23] noted an ApiAP2 (dubbed "AP2-Q"; PCYB_102390) as 446 transcriptionally up-regulated in $P$. cynomolgi hypnozoites and proposed this as a potential 447 hypnozoite marker. We note that the P. vivax ortholog of Pc-AP2-Q (PVP01_1016100) is 448 among the genes detectable as a transcript but not protein in $P$. vivax sporozoites. This may point to a translationally repressed signal in sporozoites to regulate hypnozoite formation. However, as $P v$-AP2-Q is transcribed at an abundance ( $\sim 50$ TPM) at or below which $\sim 50 \%$ of $P$. vivax genes are detectable as transcripts but not as proteins (Figure 1B), this could as likely result from LC-MS detection sensitivity. Further, although AP2-Q was reported as specific to hypnozoite forming Plasmodium species [23], it is indeed found in non-hypnozoite producing species, such as $P$. knowlesi, P. gallinaceum and $P$. inui [56]. Up-regulation of AP2-Q transcripts is not observed for hypnozoites in subsequent transcriptomic studies of $P$. cynomolgi [56] or P. vivax [22], nor do we see such an up-regulation here. Voorberg van der Wel et al [56] note transcription of a range of AP2s in P. cynomolgi liver stages, but do not find any to be up-regulated in hypnozoites. AP2s also feature among transcribed genes in $P$. vivax liver stages, with one, PVP01_0916300, significantly up-regulated in hypnozoites. We note that PVP01_0916300 is up-regulated in P. vivax sporozoites relative to blood-stages and found in the top quartile of transcripts by abundance $($ TPM $=104)$.

Chromatin epigenetics in $\boldsymbol{P}$. vivax sporozoites. As noted above, transcriptomic data for sporozoites, and their comparison with liver and blood-stages, implicate histone epigenetics as having an important role in sporozoite biology and liver stage differentiation. This concept has been alluded to in recent liver-stage studies of $P$. cynomolgi $[17,23]$ that propose methyltransferases as having a potential role in hypnozoite formation. No epigenetic data are currently available for any $P$. vivax life-cycle stage. Studies of $P$. falciparum blood-stages have identified the importance of histone modifications as a primary epigenetic regulator [57, 58] and characterized key markers of heterochromatin (H3K9me3) and euchromatin/transcriptional activation (H3K4me3 and H3K9ac). Recently, these marks have been explored with the maturation of $P$. falciparum sporozoites in the mosquito [26]. Here, we characterize major histone marks in $P$. vivax sporozoites and assess their relationship to transcript abundance.

Histone modifications in P. vivax sporozoites. Using ChIP-seq, we identified 1,506, 1,999 and 5,262 ChIP-seq peaks stably represented in multiple $P$. vivax sporozoite replicates and associated with $\mathrm{H} 3 \mathrm{~K} 9 \mathrm{me} 3, \mathrm{H} 3 \mathrm{~K} 9 \mathrm{ac}$ and $\mathrm{H} 3 \mathrm{~K} 4 \mathrm{me} 3$ histone marks respectively (Figure 4 and S14-S19). Peak width, spacing and stability differed with histone mark type (Figures S15 and S16). H3K4me3 covered the greatest breadth of the genome (36.0\% of all bases) and was the most stable among replicates, with $\sim 84 \%$ of bases associated with an H3K4me3 found in multiple biological replicates. By comparison $\mathrm{H} 3 \mathrm{~K} 9$ me3 marks were least stable, with $46 \%$ of bases associated with this mark found in just one replicate. Consistent with observations in $P$. falciparum, H3K9me3 'heterochromatin' marks primarily clustered in telomeric and subtelometric regions (Figure 4). In contrast, the 'euchromatin' / transcriptionally open histone marks, $\mathrm{H} 3 \mathrm{~K} 4 \mathrm{me} 3$ and $\mathrm{H} 3 \mathrm{~K} 9 \mathrm{ac}$, were distributed in chromosome central regions and did not overlap with regions under H3K9me3 suppression. Both H3K9me3 and H3K4me3 marks were reasonably uniformly distributed (mean peak spacing $\sim 500 \mathrm{bp}$ for each) within their respective regions of the genome. In contrast, H3K9ac peaks were spaced further apart (mean: $\sim 2 \mathrm{~kb}$ ), but also with a greater variability in spacing (likely reflecting their association with promoter regions [59]; Figure S17 and S19). The instability of H3K9me3 may reflect its use in Plasmodium for regulating expression of contingency genes from multigene families, whose members have overlapping and redundant functions [49] and confer phenotypic plasticity [60].

Genes under histone regulation. We explored an association between these histone marks and the transcriptional behaviour of protein coding genes (Figure 4 and S19 and Tables S15- 
499 ends of the chromosomal scaffolds (i.e., are (sub)telomeric). On average, these genes are

500

501

502

503

504

505

506

507

508

509

510

511

512

513

514

515

516

517

518

519

520

521

522

523

524

525

526

527

528

529

530

531

532

533

534

535

536

537

538

539

540

541

542

543

544

545

546

547

548

549

550

551

552

553 transcribed at $\sim 30$ fold lower levels (mean 0.7 TPM) than genes not stably intersected by H3K9me3 marks. These data clearly support the function of this mark in transcriptional silencing. This is largely consistent with observations in P. falciparum sporozoites [26], however, in contrast to $P$. falciparum sporozoites where a single var gene was described to lack heterochromatin structure [27] we observe no genes within heterochromatin dense region that lacked a stable $\mathrm{H} 3 \mathrm{~K} 9 \mathrm{me} 3$ signal or were transcribed at notable levels (i.e., above $\sim 5$ TPM). Whether this relates to differences in epigenetic control between the species is not clear. We note that (sub)telomeric genes are overall transcriptionally silent in $P$. vivax sporozoites relative to blood-stages (Figure 4 and Tables S21 and S22). Consistent with observations in $P$. falciparum [57], the bulk of these genes include complex protein families, such as vir and $P v$-fam genes, which are so far described to function primarily in bloodstages. Also notable among the genes are several reticulocyte-binding proteins, including RBP2, 2a, 2b and 2c. This transcriptional silence in telomeric and subtelomeric regions was recently observed in P. falciparum sporozoites [27].

Outside of the telomeres and subtelomeres, H3K4me3 marks are stably associated with the intergenic regions of 3,676 genes. $\mathrm{H} 3 \mathrm{~K} 9 \mathrm{ac}$ marks are also identified within $1 \mathrm{~kb}$ of the transcriptional start site (TSS) of 1,284 coding genes, with 1085 of these stably marked also by H3K4me3 (Figure 4B). The average transcription of these genes is 50, 112 and 112 TPMs respectively (72, 160 and 160-fold higher than H3K9me3 marked genes). Gene-bygene observations show that $\mathrm{H} 3 \mathrm{~K} 9 \mathrm{ac}$ and $\mathrm{H} 3 \mathrm{~K} 4 \mathrm{me} 3$ marks cluster densely in the $1000 \mathrm{~kb}$ up and down-stream of the start and stop codon respectively of transcribed genes, but are much less dense within coding regions of these genes (Figure S15). This pattern directly correlates with transcription and contrasts $\mathrm{H} 3 \mathrm{~K} 9 \mathrm{me} 3$ marks, which are distributed across the length of the gene at even density and are correlated with a lack of transcription. These data support the role of $\mathrm{H} 3 \mathrm{~K} 9 \mathrm{ac}$ and $\mathrm{H} 3 \mathrm{~K} 4 \mathrm{me} 3$ in transcriptional activation in $P$. vivax. The lower transcriptional abundance of $\mathrm{H} 3 \mathrm{~K} 4 \mathrm{me} 3$ marked, compared with $\mathrm{H} 3 \mathrm{~K} 9 \mathrm{ac}$ or $\mathrm{H} 3 \mathrm{~K} 9 \mathrm{ac}$ and H3K4me3 marked genes suggest these marks work synergistically and that H3K9ac is possibly the better of the two, as a single mark indicator of transcriptional activity in $P$. vivax. This is consistent with recent observations in P. falciparum sporozoites [26].

Interestingly, H3K9ac-marked genes ranged in transcriptional activity from the most abundantly transcribed genes to many in the lower 50\% and even lowest decile of transcription. This suggests more contributes to transcriptional activation in $P$. vivax sporozoites than, simply, gene accessibility through chromatin regulation. Specific activation by a transcription factor (e.g., ApiAP2s [61]) is the obvious candidate. To explore this, we compared upstream regions (within $1 \mathrm{~kb}$ of the TSS or up to the $3^{\prime}$ end of the next gene upstream, whichever was less) of highly (top 10\%) and lowly (bottom 10\%) transcribed $\mathrm{H} 3 \mathrm{~K} 9 \mathrm{ac}$ marked genes for over-represented sequence motifs in the highly expressed genes that might coincide with known ApiAP2 transcription factor binding sites [62]. We identified these based on the location of the nearest stable $\mathrm{H} 3 \mathrm{~K} 9 \mathrm{ac}$ peak relative to the transcription start site for each gene (Figure S12). In most instances, these peaks were within 100bp of the TSS and, consistent with data from P. falciparum [59], $P$. vivax promoters appear to be no more than a few hundred to a maximum of $1000 \mathrm{bp}$ upstream of the TSS. Exploring these regions, we identified two over-represented motifs: TGTACMA (e-value 2.7 $\mathrm{e}^{-2}$ ) and ATATTTH (e-value 3.3 $\mathrm{e}^{-3}$ ) (Fig. 2D). TGTAC is consistent with the known binding site for $P f$-AP2-G, which regulates sexual differentiation in gametocytes [63], but its $P$. vivax ortholog (PVP01_1418100) is neither highly transcribed nor expressed in sporozoites. ATATTTH is similar to the binding motif for $P f$-AP2-L (AATTTCC), a transcription factor that is important for liver stage development in $P$. berghei [64]. In contrast to AP2-G, $P v$ AP2-L (PVX_081180) is in the top 10\% of transcription and expression in P. vivax sporozoites and up-regulated relative to blood-stages. In P. vivax sporozoites, the ATATTTH motif is associated with a number of highly transcribed genes, including lispl and uis2-4, known to be regulated by AP2-L in P. berghei [64] as well as many of the most highly transcribed, H3K9ac marked genes, including two etramps (PVP01_0734800 and PVP01_0504800), several RNA-binding proteins, including Puf2, $d d x 5$, a putative ATP- 
554 dependent RNA helicase DBP1 (PVP01_1429700), and a putative baxl inhibitor 555 (PVP01_1465600). Interestingly, a number of highly transcribed and translationally repressed genes associated with the ATATTTH motif, including uis4, siap2 and $p v 1$, are not stably marked by $\mathrm{H} 3 \mathrm{~K} 9 \mathrm{ac}$ in all replicates (i.e., there is significant variation in the placement of the $\mathrm{H} 3 \mathrm{~K} 9 \mathrm{ac}$ peak or their presence/absence among replicates for these genes). It may be that additional histone modifications, for example H3K27me, H3R17me3 or H2A or H4 modifications, are involved in regulating transcription of these genes. Certainly the incorporation of the H2A.Z histone variant, which is present in intergenic regions of $P$. falciparum (Petter et al 2011), and controls temperature responses in plants [65] is intriguing as a potential mark regulating sporozoite fate in $P$. vivax considering the association between hypnozoite activation rate and climate [11], as is H3R17me3 in consideration of the enrichment of markers/readers of this modification in HPZs noted above and the role of this mark in cell fate progression in other species [50].

\section{CONCLUSIONS}

We provide the first comprehensive study of the transcriptome and epigenome of mature Plasmodium vivax sporozoites and undertake detailed comparisons with recently published proteomic data for $P$. vivax sporozoites [33] and transcriptomic data for $P$. vivax mixed and hypnozoite-enriched liver-stages [22] and mixed blood-stages [40]. These data support the proposal that the sporozoite is a highly-programmed stage that is primed for invasion of and development in the host hepatocyte. Cellular regulation, including at transcription, translational and epigenetic levels, appears to play a major role in shaping this stage (which continues on in some form in hypnozoites), and many of the genes proposed here as being under translational repression are involved in hepatocyte infection and early liver-stage development (Figure 5). We highlight a major role for RNA-binding proteins, including PUF2, ALBA2/4 and, intriguingly, 'Homologue of Musashi' (HoMu). We find that transcriptionally, the hypnozoite appears to be a transition point between the sporozoite and replicating schizonts, having many of the dominant sporozoite transcripts and retaining high transcription of a number of key regulatory pathways involved in transcription, translation and chromatin configuration (including histone arginine methylation). A consistent theme in the study is the prominence of a number of genes that have a role in numerous eukaryotic systems in cell fate determination and differentiation (e.g., HoMu, Yippee and CARM1) and overlap with dormancy and/or persistent cell states in bacteria, protists or higher eukaryotes (e.g., bacterial sucB and gamete fusion protein HAP2). These data do not point to one single programming switch for dormancy or liver developmental fate in $P$. vivax, but present a number of intriguing avenues for exploration in subsequent studies, particularly in model species such as $P$. cynomolgi. Our study contributes to understanding the early stages of hepatocyte infection and the developmental switch between liver trophozoite and hypnozoite formation. We also identify potential avenues for rationally prioritizing targets underpinning liver-stage differentiation for functional evaluation in humanized mouse and simian models for relapsing Plasmodium species and identifying novel avenues to understand and eradicate liver-stage infections.

\section{MATERIALS AND METHODS}

Ethics Statement. Collection of venous blood from human patients with naturally acquired vivax infection for the current study was approved by the Ethical Review Committee of the Faculty of Tropical Medicine, Mahidol University (Human Subjects Protocol number TMEC 11-033) with the informed written consent of each donor individual. All mouse tissue used in the current study was from preserved infected tissues generated previously [13]. All mouse infection work in [13] was carried out at the Centre for Infectious Diseases Research (CIDR) in Seattle, USA, under direct approval of the CIDR Institutional Animal Care and Use Committee (IACUC) and performed in strict accordance with the recommendations in the Guide for the Care and Use of Laboratory Animals of the National Institutes of Health, USA. The Centre for Infectious Disease Research Biomedical Research Institute has an Assurance 
608 from the Public Health Service (PHS Assurance number is A3640-01) through the Office of 609 Laboratory Animal Welfare (OLAW) for work approved by its IACUC.

610

611

612

613

614

615

616

617

618

619

620

621

622

623

624

625

626

627

628

629

630

631

632

633

634

635

636

637

638

639

640

641

642

643

644

645

646

647

648

649

650

651

652

653

654

655

656

657

658

659

660

661

662
Material collection, isolation and preparation. Nine field isolates (PvSpz-Thai 1 to 9), representing symptomatic blood-stage malaria infections were collected as venous blood (20 $\mathrm{mL}$ ) from patients presenting at malaria clinics in Tak and Ubon Ratchatani provinces in Thailand. Each isolate was used to establish, infections in Anopheles dirus colonized at Mahidol University (Bangkok) by membrane feeding [13], after 14-16 days post blood feeding, 3-15 million sporozoites were harvested per field isolate from the salivary glands of up to 1,000 of these mosquitoes as per [66] and shipped in preservative (trizol (RNA/DNA) or $1 \%$ paraformaldehyde (DNA for ChiP-seq) to the Walter and Eliza Hall Institute (WEHI).

Transcriptomics sequencing and differential analysis. Upon arrival at WEHI, messenger RNAs were purified from an aliquot ( $\sim 0.5-1$ million sporozoites) of each $P$. vivax field isolate as per [40] and subjected to RNA-seq on Illumina NextSeq using TruSeq library construction chemistry as per the manufacturer's instructions. Raw reads for each RNA-seq replicate are available through the Sequence Read Archive (XXX-XXX). Sequencing adaptors were removed and low quality reads trimmed and filtered using Trimmomatic v. 0.36 [67]. To remove host contaminants, processed reads were aligned, as single-end reads, to the Anopholes dirus wrari2 genome (VectorBase version W1) using Bowtie2 [68] (--verysensitive preset). All non-host reads were then aligned to the manually curated transcripts of the $P$. vivax $\mathrm{P} 01$ genome (http://www.genedb.org/Homepage/Pvivax 01 ; [28]) using RSEM [69] (pertinent settings: --bowtie2 --bowtie2-sensitivity-level very_sensitive --calc-ci --cimemory 10240 --estimate-rspd --paired-end). Transcript abundance for each gene in each replicate was calculated by RSEM as raw count, posterior mean estimate expected counts (pme-EC) and transcripts per million (TPM).

Transcriptional abundance in $P$. vivax sporozoites was compared qualitatively (by ranked abundance) with previously published microarray data for $P$. vivax salivary-gland sporozoites [24]. As a further quality control, these RNA-seq data were compared also with previously published microarray data for $P$. falciparum salivary-gland sporozoites [30], as well as RNA-seq data from salivary-gland sporozoites generated here for $P$. falciparum (single replicate generated from P. falciparum 3D7 lab cultures isolated from Anopholes stephensi and processed as above) and previously published for P. yoelii [29]. RNA-seq data from these additional Plasmodium species were (re)analysed from raw reads and transcriptional abundance for each species was determined (raw counts and pme-EC and TPM data) as described above using gene models current as of 04-10-2016 (PlasmoDB release v29). Interspecific transcriptional behaviour was qualitatively compared by relative ranked abundance in each species using TPM data for single copy orthologs (SCOs; defined in PlasmoDB) only, shared between $P$. vivax and $P$. faliciparum or shared among $P$. vivax, $P$. falciparum and $P$. yoelii.

To define transcripts that were up-regulated in sporozoites, we remapped raw reads representing early (18-24 hours post-infection (HPI)), mid (30-40 HPI) and late (42-46 HPI) $P$. vivax blood-stage infections recently published by Zhu et al [40] to the $P$. vivax $\mathrm{P} 01$ transcripts using RSEM as above. All replicate data was assessed for mapping metrics, transcript saturation and other standard QC metrics using QualiMap v 2.1.3 [70]. Differential transcription between $P$. vivax salivary-gland sporozoites and mixed blood-stages [40] was assessed using pme-EC data in EdgeR [71] and limma [72] (differential transcription cut-off: $\geq 2$-fold change in counts per million (CPM) and a False Discovery Rate (FDR) $\leq 0.05$ ). Pearson Chi squared tests were used to detect over-represented Pfam domains and Gene Ontology (GO) terms among differentially transcribed genes in sporozoites (Bonferronicorrected $p<0.05$ ), based on gene annotations in PlasmoDB (release v29).

We also compared transcription of the sporozoite stages to recently published liverstage data from Gural et al [22] as per the sporozoite to blood-stage comparisons above, with the following modifcations: (1) EC values were normalized using the 'upper quartile' method instead of TMM, (2) differential transcription was assessed using a quasi-likelihood 
663 generalize linear model (instead of a linear model) and (3) an FDR threshold for significance 664 of $\leq 0.01$ was used instead of $\leq 0.05$. These differences related to specific attributes of the

665

666

667

668

669

670

671

672

673

674

675

676

677

678

679

680

681

682

RT-qPCR Primers were as follows:

\begin{tabular}{llll} 
Name & Gene & Forward Primer & Reverse Primer \\
\hline RPS27 & PVX_122245 & ACCACCTTGTTTAGCCATGC & TAATTTGCACTTTCCACCCGTT \\
\hline D13 & PVX_089510 & CTGTACACGCACGAGCTGGC & CAGCTCCTTGACGCCACTG \\
\hline A10 & PVX_080110 & ACGAGCTGTACTACAAGCGGA & TTTCTCCTGCACCAGGTAGTC \\
\hline SERA & PVX_086995* & GCCCCACTGGAAGTTTTGGA & CGTTCAGCCGCTGGTAGTAT \\
\hline CelTOS & PVX_123510* & CCCCCAAAGGCAAAATGAACAA & CGCTCTTTCCCCTCAAGGAC \\
\hline GEST & PVX_118040 & GACATATCAAGCAGTGAGGGA & CATGTTGTGGCCTTTATATGCTG \\
\hline ALBA4 & PVX_083270 & TATCAACGGAGCCTTTGCCG & GGACTTGATTTCCTCGTCGG \\
\hline PUF2 & PVX_089945 & ATCATAGAGAACGTCGACAAGCTTA & CTACGTTTCCAGGTTGCTGATC \\
\hline 14-3-3 & PVX_089505 & GACAACTTGACCTTGTGGACGTC & TACTCGAGGCCTTCATCCTTCGATT \\
\hline ZIPCO & PVX_001980 & TTAGCTCAATTGCTTGTGGCTTTTT & TGCCACTAACTCCAAGGAAATAACT
\end{tabular}

683

684

685

686

687

688

689

690

691

692

693

694

695

696

697

698

699

700

liver-stage dataset, particularly the small number of replicates $(n=2)$ per experiment condition. Data visualization and interactive R-shiny plots were produced in $\mathrm{R}$ using the ggplot[73], ggplot2 [74], gplots(heatmap.2) [75] and Glimma [76] packages.

Assessment of Sporozoite RNA-seq transcriptome by selective RT-qPCR: Extracted RNA was DNase treated (Sigma D5307) as per manufacturer recommendations. RNA was quantified using the TapeStation High Sensitivity RNA kit (Agilent). Two intron-spanning primer pairs were designed per gene of interest using Primer3 and BLAST. Primer pairs were tested in two concentrations $(0.75 \mathrm{ng}$ and $2.83 \mathrm{ng}$ per reaction) to determine efficiency and specificity. Product was run on a $1 \%$ agarose gel with ethidium bromide. Primer pairs indicating non-specific priming were removed. The resulting 11 primer pairs were used on four sporozoite samples; VUBR06, VUNL23, VUBR24, VTTY84. RNA was reverse transcribed (Sensifast, Bioline) and used at $0.75 \mathrm{ng}$ per reaction, run on a Roche LightCycler 480 II. Melt curves were assessed and products were run on a gel to ensure specificity again. $\mathrm{Cp}$ threshold was set automatically. $\Delta \mathrm{Cp}$ value was calculated as target gene - comparator gene (SERA and CelTOS were used). Data were log transformed and fold change calculated.

* denotes single exon gene

Salivary-gland sporozoite and liver-stage immunofluorescence assays (IFAs). IFAs were performed as per [13] using preserved, vivax infected mouse liver tissue generated previously for that study. In [13], female FRG [fumarylacetoacetate hydrolase (F), recombination activation gene $2(\mathrm{R})$, interleukin-2 receptor subunit gamma $(\mathrm{G})]$ triple KO mice engrafted with human hepatocytes (FRG KO huHep) were purchased from Yecuris Corporation (Oregon, USA). Mice were infected through intravenous injection into the tail with $3.5 \times$ $10^{5}$ to $1 \times 10^{6}$ sporozoites isolated from the salivary glands of infected mosquitoes in $100 \mu \mathrm{l}$ of RPMI media. Liver stages for the current study were obtained from $10 \mu \mathrm{m}$ formalin fixed paraffin embedded day 7 liver stages generated previously [13] from FRG knockout huHep mice;[13] these were deparaffinized prior to staining. Fresh salivary-gland sporozoites were fixed in acetone per [13]. All cells were incubated twice for 3 minutes in Xylene, then 100\% Ethanol, and finally once for 3 minutes each in 95\%, 70\%, and 50\% Ethanol. The cells were rinsed in DI water and permeabilized immediately in 1XTBS, containing Triton X-100 and $30 \%$ hydrogen peroxide. The cells were blocked in 5\% milk in 1XTBS. The hepatocytes were stained overnight with a rabbit polyclonal LISP1 antibody (A), a rabbit polyclonal UIS4 
701 antibody (B), and a rabbit polyclonal BIP antibody (C) in blocking buffer. The cells were washed with 1 XTBS and the primary antibodies were detected with goat anti-rabbit Alexa Fluor 488 antibody (Life Technologies). The cells were washed in 1XTBS. The hepatocytes were rinsed in KMNO4 and washed in 1XTBS. The cells were incubated in DAPI for 5 minutes.

Histone ChIP sequencing and analysis. Aliquots of $2-6$ million freshly isolated sporozoites were fixed with $1 \%$ paraformaldehyde for $10 \mathrm{~min}$ at $37^{\circ} \mathrm{C}$ and the reaction subsequently quenched by adding glycine to a final concentration of $125 \mathrm{mM}$. After three washes with PBS, sporozoite pellets were stored at $-80^{\circ} \mathrm{C}$ and shipped to Australia. Nuclei were released from the sporozoites by dounce homogenization in lysis buffer $(10 \mathrm{mM}$ Hepes pH 7.9, $10 \mathrm{mM} \mathrm{KCl}, 0.1 \mathrm{mM}$ EDTA, $0.1 \mathrm{mM}$ EDTA, $1 \mathrm{mM}$ DTT, 1x EDTA-free protease inhibitor cocktail (Roche), $0.25 \%$ NP40). Nuclei were pelleted by centrifugation at 21,000 g for $10 \mathrm{~min}$ at $4^{\circ} \mathrm{C}$ and resuspended in SDS lysis buffer (1\% SDS, $10 \mathrm{mM}$ EDTA, $50 \mathrm{mM}$ Tris pH 8.1, 1x EDTA-free protease inhibitor cocktail). Chromatin was sheared into 200-1000 bp fragments by sonication for 16 cycles in $30 \mathrm{sec}$ intervals (on/off, high setting) using a Bioruptor (Diagenode) and diluted 1:10 in ChIP dilution buffer (0.01\% SDS, $1.1 \%$ Triton X100, $1.2 \mathrm{mM}$ EDTA, $16.7 \mathrm{mM}$ Tris $\mathrm{pH} 8.1,150 \mathrm{mM} \mathrm{NaCl})$. Chromatin was precleared for 1 hour with protein $\mathrm{A} / \mathrm{G}$ sepharose (4FastFlow, GE Healthcare) equilibrated in $0.1 \%$ BSA (Sigma-Aldrich, USA) in ChIP dilution buffer. Chromatin from $3 \times 10^{5}$ nuclei was taken aside as input material. Chromatin from approximately $3 \times 10^{6}$ sporozoite nuclei was used for each ChIP. ChIP was carried out over night at $4^{\circ} \mathrm{C}$ with $5 \mu \mathrm{g}$ of antibody (H3 K9me3 (Active Motif), H3K4me3 (Abcam), H3K9ac (Upstate), H4K16ac (Abcam)) and $10 \mu 1$ each of equilibrated protein A and G sepharose beads (4FastFlow, GE Healthcare). After washes in low-salt, high-salt, LiCl, and TE buffers (EZ-ChIP Kit, Millipore), precipitated complexes were eluted in $1 \%$ SDS, $0.1 \mathrm{M} \mathrm{NaHCO}_{3}$. Cross-linking of the immune complexes and input material was reversed for 6 hours at $45^{\circ} \mathrm{C}$ after addition of $500 \mathrm{mM} \mathrm{NaCl}$ and $20 \mu \mathrm{g} / \mathrm{ml}$ of proteinase K (NEB). DNA was purified using the MinElute ${ }^{\circledR}$ PCR purification kit (Qiagen) and paired-end sequenced on Illumina NextSeq using TruSeq library construction chemistry as per the manufacturer's instructions. Raw reads for each ChIP-seq replicate are available through the Sequence Read Archive (XXX-XXX).

Fastq files were checked for quality using fastqc (http://www.bioinformatics.babraham.ac.uk/projects/fastqc/) and adapter sequences were trimmed using cutadapt [77]. Paired end reads were mapped to the P. vivax P01 strain genome annotation using Bowtie2 [68]. The alignment files were converted to Bam format, sorted and indexed using Samtools [78]. ChIP peaks were called relative to input using MACS2[79] in paired end mode with a q value less than or equal to 0.01. Peaks and peak summits were converted to sorted BED files. Bedtools intersect[80] was used to identify genes that intersected $\mathrm{H} 3 \mathrm{~K} 9$ me3 peaks and Bedtools closest was used to identify genes that were closest to and downstream of $\mathrm{H} 3 \mathrm{~K} 9 \mathrm{ac}$ and $\mathrm{H} 3 \mathrm{~K} 4 \mathrm{me} 3$ peak summits.

Sequence motif analysis. Conserved sequence motifs were identified using the program DREME [81]. Only genes in the top decile of transcription showing no evidence of protein expression in multiple salivary-gland sporozoite replicates were considered as putatively translationally repressed $(n=170)$. We queried coding regions and regions upstream of the transcriptional start site (TSS) for each gene, defined by Zhu et al [40] and/or predicted here from all RNA-seq data using the Tuxedo suite [82], for enriched sequence motifs in comparison to 170 genes found to be in the top decile of both transcriptional and expressional abundance in the same sporozoite replicates. In searching for motifs associated with highly transcribed genes with stable H3K9ac marks within $1 \mathrm{~kb}$ of the TSS (or up to the 3' end of the next gene upstream), we compared $\mathrm{H} 3 \mathrm{~K} 9 \mathrm{ac}$ marked genes in the top decile of transcription to the same number of $\mathrm{H} 3 \mathrm{~K} 9 \mathrm{ac}$ marked genes in the bottom decile of transcription. In both instances, an e-value threshold of 0.05 was considered the minimum threshold for statistical significance. 


\section{References}

757 1. Organization WH. World Malaria Report 2015. WHO, Geneva. 2015.

758 2. Feachem RG, Phillips AA, Hwang J, Cotter C, Wielgosz B, Greenwood BM, et al.

759 Shrinking the malaria map: progress and prospects. Lancet. 2010;376

760

761

762

763

764

3. Price RN, Douglas NM and Anstey NM. New developments in Plasmodium vivax malaria: severe disease and the rise of chloroquine resistance. Curr Opin Infect Dis. 2009;22 5:430-5. doi:10.1097/QC0.0b013e32832f14c1.

4. Baird KJ. Malaria caused by Plasmodium vivax: recurrent, difficult to treat, disabling, and threatening to life - averting the infectious bite preempts these hazards. Pathogens and global health. 2013;107:475-9.

5. Sattabongkot J, Tsuboi T, Zollner GE, Sirichaisinthop J and Cui L. Plasmodium vivax transmission: chances for control? Trends in parasitology. 2004;20 4:1928.

6. Mueller I, Galinski MR, Baird JK, Carlton JM, Kochar DK, Alonso PL, et al. Key gaps in the knowledge of Plasmodium vivax, a neglected human malaria parasite. Lancet Infect Dis. 2009;9 9:555-66.

7. Lindner SE, Miller JL and Kappe SH. Malaria parasite pre-erythrocytic infection: preparation meets opportunity. Cell Microbiol. 2012;14 3:316-24.

8. Shin SC, Vanderberg JP and Terzakis JA. Direct infection of hepatocytes by sporozoites of Plasmodium berghei. J Protozool. 1982;29 3:448-54.

9. Mota MM, Pradel G, Vanderberg JP, Hafalla JC, Frevert U, Nussenzweig RS, et al. Migration of Plasmodium sporozoites through cells before infection. Science. 2001;291 5501:141-4.

10. Lysenko AJ, Beljaev A and Rybalka V. Population studies of Plasmodium vivax: 1. The theory of polymorphism of sporozoites and epidemiological phenomena of tertian malaria. Bull WHO. 1977;55 5:541.

11. White NJ. Determinants of relapse periodicity in Plasmodium vivax malaria. Malar J. 2011;10:297.

12. Price RN, Tjitra E, Guerra CA, Yeung S, White NJ and Anstey NM. Vivax malaria: neglected and not benign. Amer J Trop Med Hyg. 2007;77 6 Suppl:79-87.

13. Mikolajczak SA, Vaughan AM, Kangwanrangsan N, Roobsoong W, Fishbaugher M, Yimamnuaychok $\mathrm{N}$, et al. Plasmodium vivax liver stage development and hypnozoite persistence in human liver-chimeric mice. Cell Host Microbe. 2015;17 4:526-35.

14. Mueller A-K, Camargo N, Kaiser K, Andorfer C, Frevert U, Matuschewski K, et al. Plasmodium liver stage developmental arrest by depletion of a protein at the parasite-host interface. Proc Nat'l Acad Sci USA. 2005;102 8:3022-7.

15. Silvie O, Briquet S, Muller K, Manzoni G and Matuschewski K. Posttranscriptional silencing of UIS4 in Plasmodium berghei sporozoites is important for host switch. Mol Microbiol. 2014;91 6:1200-13.

16. Mackellar DC, O'Neill MT, Aly AS, Sacci JB, Jr., Cowman AF and Kappe SH. Plasmodium falciparum PF10_0164 (ETRAMP10.3) is an essential parasitophorous vacuole and exported protein in blood stages. Eukaryot Cell. 2010;9 5:784-94.

17. Dembele L, Franetich JF, Lorthiois A, Gego A, Zeeman AM, Kocken CH, et al. Persistence and activation of malaria hypnozoites in long-term primary hepatocyte cultures. Nat Med. 2014;20 3:307-12.

18. Malmquist NA, Moss TA, Mecheri S, Scherf A and Fuchter MJ. Small-molecule histone methyltransferase inhibitors display rapid antimalarial activity against all blood stage forms in Plasmodium falciparum. Proc Nat'l Acad Sci USA. 2012;109 41:16708-13.

19. Josling GA and Llinas M. Sexual development in Plasmodium parasites: knowing when it's time to commit. Nat Rev Microbiol. 2015;13 9:573-87. 
810

811

812

813

814

815

816

817

818

819

820

821

822

823

824

825

826

827

828

829

830

831

832

833

834

835

836

837

838

839

840

841

842

843

844

845

846

847

848

849

850

851

852

853

854

855

856

857

858

859

860

861

862

863
20. White MT, Karl S, Battle KE, Hay SI, Mueller I and Ghani AC. Modelling the contribution of the hypnozoite reservoir to Plasmodium vivax transmission. Elife. $2014 ; 3$.

21. Roobsoong W, Tharinjaroen CS, Rachaphaew N, Chobson P, Schofield L, Cui L, et al. Improvement of culture conditions for long-term in vitro culture of Plasmodium vivax. Malaria J. 2015;14 1:1.

22. Gural N, Mancio-Silva L, Miller AB, Galstian A, Butty VL, Levine SS, et al. In vitro culture, drug sensitivity, and transcriptome of Plasmodium vivax hypnozoites. Cell Host Microbe. 2018;23 3:395-406 e4.

23. Cubi R, Vembar SS, Biton A, Franetich JF, Bordessoulles M, Sossau D, et al. Laser capture microdissection enables transcriptomic analysis of dividing and quiescent liver stages of Plasmodium relapsing species. Cell Microbiol. 2017.

24. Westenberger SJ, McClean CM, Chattopadhyay R, Dharia NV, Carlton JM, Barnwell JW, et al. A systems-based analysis of Plasmodium vivax lifecycle transcription from human to mosquito. PLoS Negl Trop Dis. 2010;4 4:e653.

25. Kim A, Popovici J, Vantaux A, Samreth R, Bin S, Kim S, et al. Characterization of P. vivax blood stage transcriptomes from field isolates reveals similarities among infections and complex gene isoforms. Sci Rep. 2017;7 1:7761.

26. Gomez-Diaz E, Yerbanga RS, Lefevre T, Cohuet A, Rowley MJ, Ouedraogo JB, et al. Epigenetic regulation of Plasmodium falciparum clonally variant gene expression during development in Anopheles gambiae. Sci Rep. 2017;7:40655.

27. Zanghi G, Vembar SS, Baumgarten S, Ding S, Guizetti J, Bryant JM, et al. A specific PfEMP1 is expressed in P. falciparum sporozoites and plays a role in hepatocyte infection. Cell Rep. 2018;22 11:2951-63.

28. Auburn S, Bohme U, Steinbiss S, Trimarsanto H, Hostetler J, Sanders M, et al. A new Plasmodium vivax reference sequence with improved assembly of the subtelomeres reveals an abundance of pir genes. Wellcome Open Res. 2016;1:4.

29. Lindner SE, Mikolajczak SA, Vaughan AM, Moon W, Joyce BR, Sullivan WJ, Jr., et al. Perturbations of Plasmodium Puf2 expression and RNA-seq of Puf2-deficient sporozoites reveal a critical role in maintaining RNA homeostasis and parasite transmissibility. Cell Microbiol. 2013;15 7:1266-83.

30. Le Roch KG, Johnson JR, Florens L, Zhou Y, Santrosyan A, Grainger M, et al. Global analysis of transcript and protein levels across the Plasmodium falciparum life cycle. Genome research. 2004;14 11:2308-18.

31. Mikolajczak SA, Silva-Rivera H, Peng X, Tarun AS, Camargo N, Jacobs-Lorena V, et al. Distinct malaria parasite sporozoites reveal transcriptional changes that cause differential tissue infection competence in the mosquito vector and mammalian host. Mol Cell Biol. 2008;28 20:6196-207.

32. Carlton JM, Adams JH, Silva JC, Bidwell SL, Lorenzi H, Caler E, et al. Comparative genomics of the neglected human malaria parasite Plasmodium vivax. Nature. 2008;455 7214:757-63.

33. Swearingen KE, Lindner SE, Flannery EL, Vaughan AM, Morrison RD, Patrapuvich R, et al. Proteogenomic analysis of the total and surface-exposed proteomes of Plasmodium vivax salivary gland sporozoites. PLoS Negl Trop Dis. 2017;11 7:e0005791.

34. Guerreiro A, Deligianni E, Santos JM, Silva PA, Louis C, Pain A, et al. Genome-wide RIP-Chip analysis of translational repressor-bound mRNAs in the Plasmodium gametocyte. Genome Biol. 2014;15 11:493.

35. Silvie O, Briquet $\mathrm{S}$, Müller $\mathrm{K}$, Manzoni $\mathrm{G}$ and Matuschewski K. Post-transcriptional silencing of UIS4 in Plasmodium berghei sporozoites is important for host switch. Molecular microbiology. 2014;91 6:1200-13.

36. Lindner SE, Swearingen KE, Harupa A, Vaughan AM, Sinnis P, Moritz RL, et al. Total and putative surface proteomics of malaria parasite salivary gland sporozoites. Mol Cell Proteomics : MCP. 2013;12 5:1127-43. 
864 37. Kelley KD, Miller KR, Todd A, Kelley AR, Tuttle R and Berberich SJ. YPEL3, a p53-

865

866

867

868

869

870

871

872

873

874

875

876

877

878

879

880

881

882

883

884

885

886

887

888

889

890

891

892

893

894

895

896

897

898

899

900

901

902

903

904

905

906

907

908

909

910

911

912

913

914

915

916 regulated gene that induces cellular senescence. Cancer Res. 2010;70 9:3566-75.

38. Tuttle R, Simon M, Hitch DC, Maiorano JN, Hellan M, Ouellette J, et al. Senescenceassociated gene YPEL3 is downregulated in human colon tumors. Ann Surg Oncol. 2011;18 6:1791-6.

39. Roth A, Adapa SR, Zhang M, Liao X, Saxena V, Goffe R, et al. Unraveling the Plasmodium vivax sporozoite transcriptional journey from mosquito vector to human host. Sci Rep. 2018;8 1:12183.

40. Zhu L, Mok S, Imwong M, Jaidee A, Russell B, Nosten F, et al. New insights into the Plasmodium vivax transcriptome using RNA-Seq. Sci Rep. 2016;6:20498.

41. Kramer S. RNA in development: how ribonucleoprotein granules regulate the life cycles of pathogenic protozoa. WIR: RNA. 2014;5 2:263-84.

42. Tucker RP. The thrombospondin type 1 repeat superfamily. Int J Biochem Cell Biol. 2004;36 6:969-74.

43. Ntumngia FB, Bouyou-Akotet MK, Uhlemann AC, Mordmuller B, Kremsner PG and Kun JF. Characterisation of a tryptophan-rich Plasmodium falciparum antigen associated with merozoites. Mol Biochem Parasitol. 2004;137 2:349-53.

44. Gubbels MJ, Vaishnava S, Boot N, Dubremetz JF and Striepen B. A MORN-repeat protein is a dynamic component of the Toxoplasma gondii cell division apparatus. J Cell Sci. 2006;119 Pt 11:2236-45.

45. Aly AS, Lindner SE, MacKellar DC, Peng X and Kappe SH. SAP1 is a critical posttranscriptional regulator of infectivity in malaria parasite sporozoite stages. Mol Microbiol. 2011;79 4:929-39.

46. Okano H, Imai T and Okabe M. Musashi: a translational regulator of cell fate. Journal of Cell Science. 2002;115 7:1355-9.

47. Cui L, Lindner S and Miao J. Translational regulation during stage transitions in malaria parasites. Annals N Y Acad Sci. 2015;1342 1:1-9.

48. Lasko P. Gene regulation at the RNA layer: RNA binding proteins in intercellular signaling networks. Sci STKE. 2003;179:RE6.

49. Guizetti J and Scherf A. Silence, activate, poise and switch! Mechanisms of antigenic variation in Plasmodium falciparum. Cell microbiol. 2013;15 5:718-26.

50. Wu Q, Bruce AW, Jedrusik A, Ellis PD, Andrews RM, Langford CF, et al. CARM1 is required in embryonic stem cells to maintain pluripotency and resist differentiation. Stem Cells. 2009;27 11:2637-45.

51. Shi S and Ehrt S. Dihydrolipoamide acyltransferase is critical for Mycobacterium tuberculosis pathogenesis. Infect Immun. 2006;74 1:56-63.

52. Ma C, Sim S, Shi W, Du L, Xing D and Zhang Y. Energy production genes sucB and ubiF are involved in persister survival and tolerance to multiple antibiotics and stresses in Escherichia coli. FEMS Microbiol Lett. 2010;303 1:33-40.

53. Schrader J, Moyle R, Bhalerao R, Hertzberg M, Lundeberg J, Nilsson P, et al. Cambial meristem dormancy in trees involves extensive remodelling of the transcriptome. Plant J. 2004;40 2:173-87.

54. Yazawa $\mathrm{K}$ and Kamada $\mathrm{H}$. Identification and characterization of carrot HAP factors that form a complex with the embryo-specific transcription factor CLEC1. J Exp Bot. 2007;58 13:3819-28.

55. Wood FC, Heidari A and Tekle YI. Genetic Evidence for Sexuality in Cochliopodium (Amoebozoa). J Hered. 2017;108 7:769-79.

56. Voorberg-van der Wel A, Roma G, Gupta DK, Schuierer S, Nigsch F, Carbone W, et al. A comparative transcriptomic analys is of replicating and dormant liver stages of the relapsing malaria parasite Plasmodium cynomolgi. Elife. 2017;6

57. Lopez-Rubio J-J, Mancio-Silva L and Scherf A. Genome-wide analysis of heterochromatin associates clonally variant gene regulation with perinuclear repressive centers in malaria parasites. Cell host \& microbe. 2009;5 2:179-90. 
917 58. Duffy MF, Selvarajah SA, Josling GA and Petter M. Epigenetic regulation of the

918

919

920

921

922

923

924

925

926

927

928

929

930

931

932

933

934

935

936

937

938

939

940

941

942

943

944

945

946

947

948

949

950

951

952

953

954

955

956

957

958

959

960

961

962

963

964

965

966

967

968

969

970

Plasmodium falciparum genome. Brief Funct Genomics. 2014;13 3:203-16.

59. Cui L, Miao J, Furuya T, Li X, Su XZ and Cui L. PfGCN5-mediated histone H3 acetylation plays a key role in gene expression in Plasmodium falciparum. Eukaryot Cell. 2007;6 7:1219-27.

60. Rovira-Graells N, Gupta AP, Planet E, Crowley VM, Mok S, de Pouplana LR, et al. Transcriptional variation in the malaria parasite Plasmodium falciparum. Genome research. 2012;22 5:925-38.

61. De Silva EK, Gehrke AR, Olszewski K, León I, Chahal JS, Bulyk ML, et al. Specific DNA-binding by apicomplexan AP2 transcription factors. Proc Nat'l Acad Sci. 2008;105 24:8393-8.

62. Painter HJ, Campbell TL and Llinás M. The Apicomplexan AP2 family: integral factors regulating Plasmodium development. Mol Biochem Parasitol. 2011;176 1:1-7.

63. Kafsack BF, Rovira-Graells N, Clark TG, Bancells C, Crowley VM, Campino SG, et al. A transcriptional switch underlies commitment to sexual development in human malaria parasites. Nature. 2014;507 7491:248.

64. Iwanaga S, Kaneko I, Kato T and Yuda M. Identification of an AP2-family protein that is critical for malaria liver stage development. PLoS One. 2012;7 11:e47557.

65. Boden SA, Kavanova M, Finnegan EJ and Wigge PA. Thermal stress effects on grain yield in Brachypodium distachyon occur via H2A.Z-nucleosomes. Genome Biol. 2013;14 6:R65.

66. Kennedy M, Fishbaugher ME, Vaughan AM, Patrapuvich R, Boonhok R, Yimamnuaychok $\mathrm{N}$, et al. A rapid and scalable density gradient purification method for Plasmodium sporozoites. Malar J. 2012;11:421.

67. Bolger AM, Lohse $M$ and Usadel B. Trimmomatic: a flexible trimmer for Illumina sequence data. Bioinformatics (Oxford, England). 2014;30 15:2114-20.

68. Langmead B and Salzberg SL. Fast gapped-read alignment with Bowtie 2. Nat Methods. 2012;9 4:357-9.

69. Li B and Dewey CN. RSEM: accurate transcript quantification from RNA-Seq data with or without a reference genome. BMC bioinformatics. 2011;12 1:323.

70. Okonechnikov K, Conesa A and Garcia-Alcalde F. Qualimap 2: advanced multisample quality control for high-throughput sequencing data. Bioinformatics (Oxford, England). 2016;32 2:292-4.

71. Nikolayeva $O$ and Robinson MD. edgeR for differential RNA-seq and ChIP-seq analysis: an application to stem cell biology. Methods Mol Biol. 2014;1150:4579.

72. Ritchie ME, Phipson B, Wu D, Hu Y, Law CW, Shi W, et al. limma powers differential expression analyses for RNA-sequencing and microarray studies. Nucleic Acids Res. 2015;43 7:e47-e.

73. Wickham H. ggplot: An implementation of the Grammar of Graphics in R, 2006. R package version 040 .

74. Wickham $\mathrm{H}$ and Chang W. ggplot2: An implementation of the Grammar of Graphics. R package version 07, URL: http://CRAN R-project org/package= ggplot2. 2008.

75. Warnes GR, Bolker B, Bonebakker L, Gentleman R, Huber W, Liaw A, et al. gplots: Various R programming tools for plotting data. R package version. 2009;2 4:1.

76. Law CW, Alhamdoosh M, Su S, Smyth GK and Ritchie ME. RNA-seq analysis is easy as 1-2-3 with limma, Glimma and edgeR. F1000Research. 2016;5.

77. Martin M. Cutadapt removes adapter sequences from high-throughput sequencing reads. EMBnet journal. 2011;17 1:pp. 10-2.

78. Li H, Handsaker B, Wysoker A, Fennell T, Ruan J, Homer N, et al. The Sequence Alignment/Map format and SAMtools. Bioinformatics (Oxford, England). 2009;25 16:2078-9. 
79. Zhang Y, Liu T, Meyer CA, Eeckhoute J, Johnson DS, Bernstein BE, et al. Modelbased analysis of ChIP-Seq (MACS). Genome Biol. 2008;9 9:R137.

80. Quinlan AR and Hall IM. BEDTools: a flexible suite of utilities for comparing genomic features. Bioinformatics (Oxford, England). 2010;26 6:841-2.

81. Bailey TL. DREME: motif discovery in transcription factor ChIP-seq data. Bioinformatics (Oxford, England). 2011;27 12:1653-9.

82. Trapnell C, Roberts A, Goff L, Pertea G, Kim D, Kelley DR, et al. Differential gene and transcript expression analysis of RNA-seq experiments with TopHat and Cufflinks. Nat Protoc. 2012;7 3:562-78.

\section{Figure Legends:}

Fig. 1 Transcriptional activity of the P. vivax sporozoite and evidence for translational repression. a Relative transcript abundance of key marker genes for sporozoites inferred by RNA-seq and qPCR (here) relative to previously published microarray data [24]; b Relative proportion of genes detectable as transcripts and proteins or transcripts only in RNA-seq and previously published proteomic data. Dashed line shows cut-off used in the current study for putatively repressed transcripts. Immunofluorescent staining of select proteins either known (UIS4) or predicted here (LISP1, EXP1 and ACP) to be translationally repressed in sporozoites in $\mathbf{c}$ sporozoite stages. CSP, mTIP as known positive controls and TRAP and BIP as exerpimental positive controls and $\mathbf{d}$ liver stages (schizonts) at 7 days post-infection in HuHep mice. Liver expression of EXP1 and ACP has been demonstrated by IFA in Mikolajczak et al [13], using the same antibodies as used here.

Fig. 2 Differential transcription between Plasmodium vivax salivary-gland sporozoites and blood-stages. A BCV plot showing separation between blood-stage (black) and salivary-gland sporozoite (red) biological replicates. B Volcano plot of distribution of fold-changes (FC) in transcription between blood-stages and salivary-gland sporozoites relative to statistical significance threshold (False Discovery Rate $($ FDR $) \leq 0.05$ ). Positive FC represents upregulated transcription in the sporozoite stage. C Mirror plot showing pFam domains statistically significantly (FDR $\leq 0.05$ ) over-represented in salivary-gland sporozoite upregulated (red) or blood-stage up-regulated (black) transcripts. Scale bar truncated for presentation. * - 55 PRESAN domains are in this dataset. ** - 99 Vir domains are in this dataset.

Fig. 3 Differential transcription between P. vivax sporozoites (SPZ), mixed (mLS) and hypnozoite (HPZ) enriched liver stages (liver-stage data from Gural et al [22]). A Heatmap comparisons showing summed transcription of enriched Pfam domains in HPZ vs mLS (left), SPZ vs HPZ (top middle) and SPZ vs mLS (top right) comparisons. All Pfam domains statistically significantly enriched at p-value 0.05). All transcript data for stage up-regulated genes at FDR 0.01). B Violin box-plot showing relative fold-change differences between SPZ and HPZ compared with SPZ and mLS for genes down-regulated in mLS compared to SPZ, but not down-regulated in HPZ compared to SPZ. C Ternary heatmap summarizing relative transcript abundance in each of SPZ, mLS and HPZ stages.

Fig. 4 Histone epigenetics relative to transcriptional behaviour in salivary-gland sporozoites. a Representative $\mathrm{H} 3 \mathrm{~K} 9 \mathrm{me} 3, \mathrm{H} 3 \mathrm{~K} 4 \mathrm{me} 3$ and $\mathrm{H} 3 \mathrm{~K} 9 \mathrm{ac}$ ChIP-seq data (grey) from a representative chromosome $(P$. vivax $\mathrm{P} 01 \mathrm{Chr}$ ) relative to mRNA transcription in salivarygland sporozoites (black) and blood-stages (black). Small numbers to top left of each row show data range. b Salivary-gland sporozoite transcription relative to nearest stable histone epigenetic marks. Numbers at the top of the figure represent total genes included in each category. Numbers within in box plot represent mean transcription in transcripts per million (TPM). c Sequence motifs enriched within $1 \mathrm{~kb}$ upstream of the Transcription Start Site of highly transcribed (top 10\%) relative to lowly transcribed genes associated with $\mathrm{H} 3 \mathrm{~K} 9 \mathrm{ac}$ marks in salivary-gland sporozoites. d Relative transcription of (sub)telomeric genes in $P$. vivax salivary-gland sporozoites and blood-stages categorized by gene sets up-regulated in 
1025 blood-stages (blue), salivary sporozoites (red) or not stage enriched (grey). Numbers in each 1026 box show mean transcription in TPM.

1027

1028

1029

1030

1031

1032

1033

1034

1035
Fig. 5 Schematic of potential mechanisms underpinning development in differentiation of $P$. vivax sporozoites during liver-stage infection as hypnozoites and schizonts. We suggest differentiation programming at different points in development; first, schizont or hypnozoite fate possibly encoded in the sporozoite as epigenetic signals or translationally repressed transcripts; secondly, suppression signals that halt progression of the hypnozoite to schizont stage and support persistence; and finally activation signals signified by a release in chromatin, (post)transcriptional and (post)translational control leading to up-regulation of replication, metabolic and protein export pathways. 
1

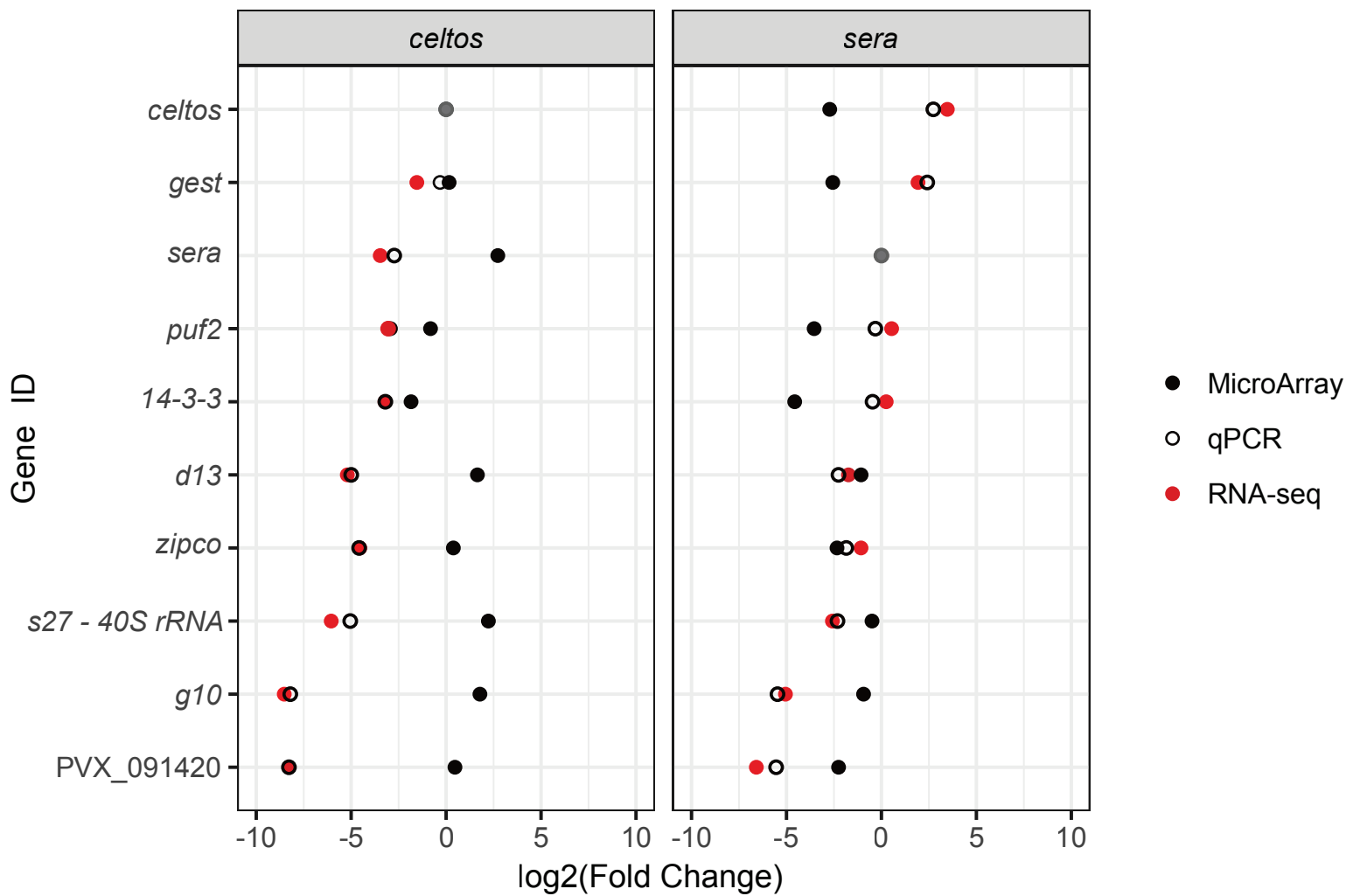

B

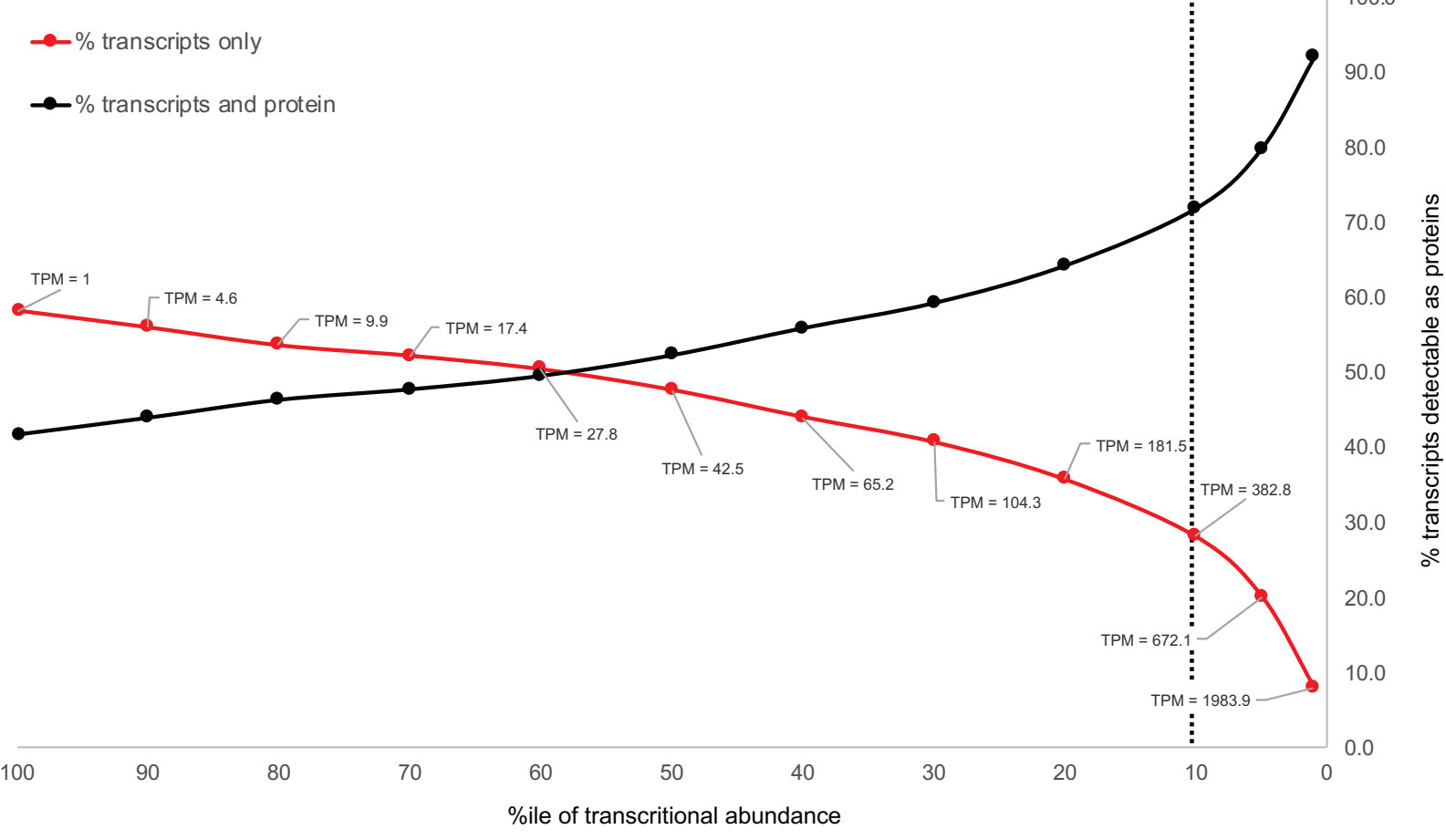

C
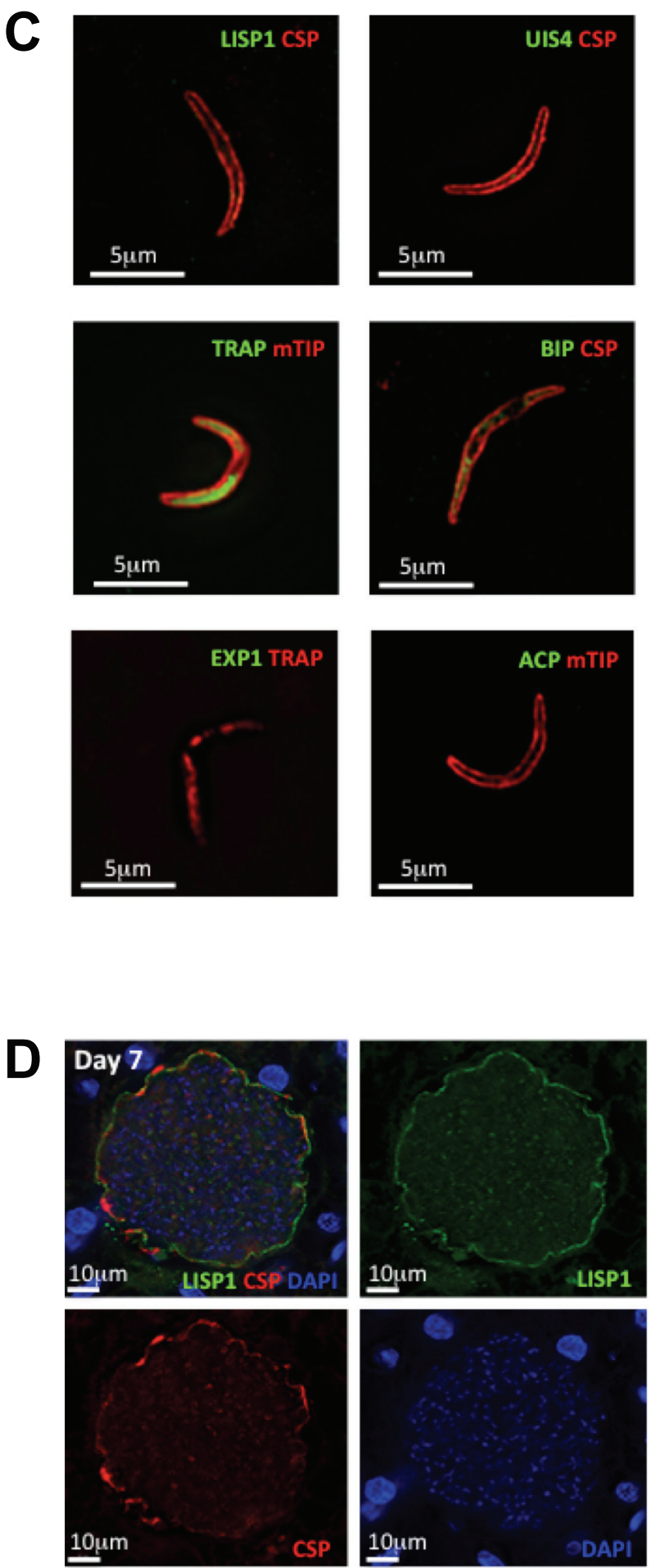
A
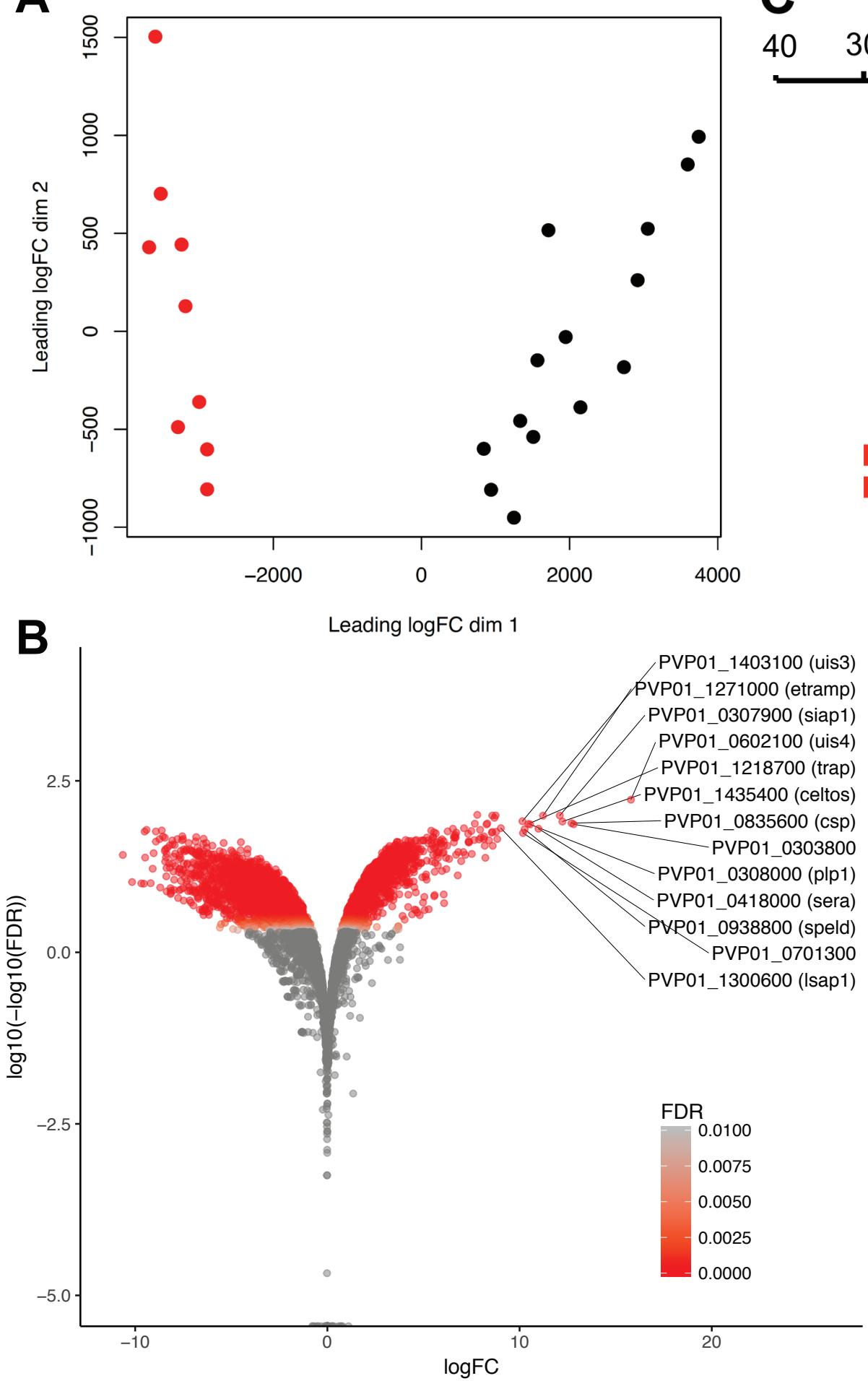

Sporozoite

$\begin{array}{lllll}40 & 30 & 20 & 10 & 0\end{array}$

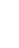

PF00076: tRNA-synt 1

PF00271: TSP-1

PF00227: VWA

PF00270: Metallophos

PF07422: zf-C3HC4_2

PF00112: s48-45

PF03815: cNMP-binding

PF09688: C2

PF12420: Bromodomain

PF00125: PI3-PI4 kinase

PF02493: EF-hand 5

PF05795: RRM-1

PF09687: Helicase C

PF08238: RRM_6

PF12319: DEAD

PF09717: MORN

PF00118: PseudoU synth-1

PF03028: PRESAN

- PF13499: Plasmodium_Vir

PF13499: Sel1

I PF00152: ABC membrane

I PF10584: Pro-isomerase

PF12774: Peptidase C1

- PF00160: Cpn60 TCP1

PF03561: TryThrA-C

PF05217: CPW-WPC

PF08393: Proteasome

PF12948: LCCL

PF13894: Wx5-PLAF3D7

PF00149: DUF3671

PF00133: Histone

PF00090: Dynein_heavy

PF00092: tRNA-synt_2

PF13923: Proteasome A-N

PF14259: AAA-6

PF00027: Allantoicase

PF00168: STOP

PF00439: DHC-N2

PF01416: MSP7-C

PF00454: zf-C2H2-4
Blood-stage

$\begin{array}{lllll}0 & 10 & 20 & 30 & 40\end{array}$
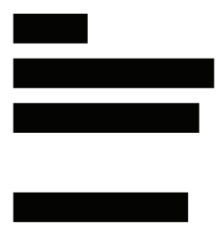

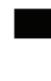
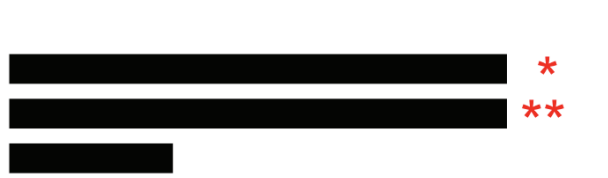


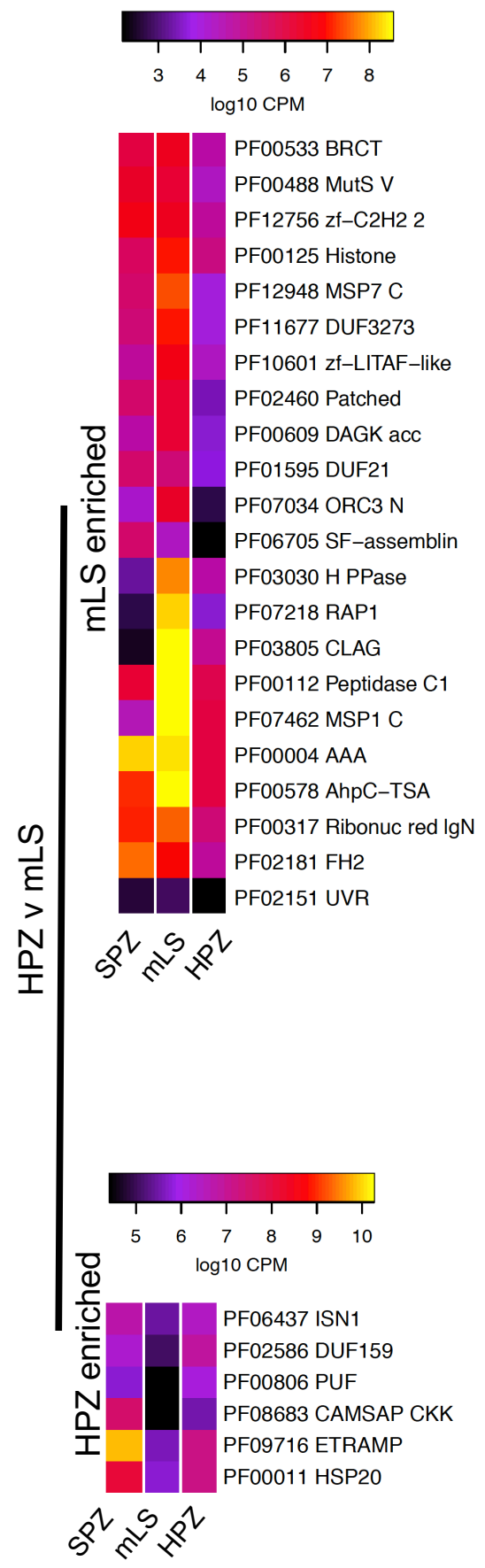

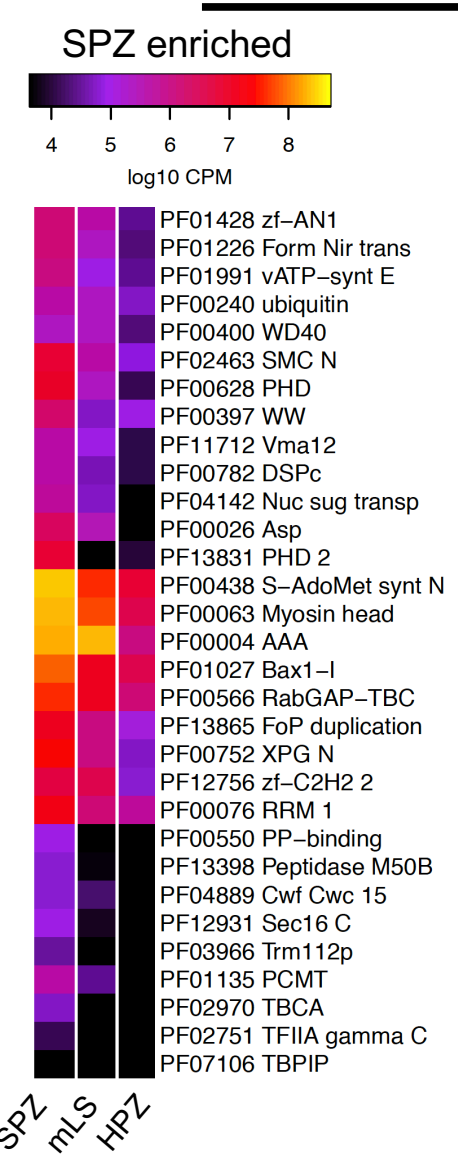

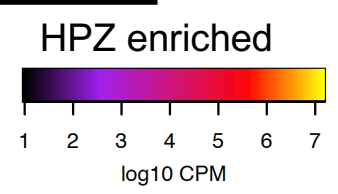

PF01398 JAB PF00467 KOW PF01694 Rhomboid PF01694 Rhom
PF00996 GDI PF00380 Ribosomal S9 PF00227 Proteasome PF00118 Cpn60 TCP1 PF00112 Peptidase C1 PF04857 CAF1

PF00268 Ribonuc red sm PF03805 CLAG

PF01612 DNA pol A exo1 PF01138 RNase PH PF00464 SHMT PF04051 TRAPP

PF00676 E1 dh

PF01066 CDP-OH P transf PF03981 Ubiq cyt C chap PF01214 CK II beta PF01458 UPF0051 PF02779 Transket pyr PF01370 Epimerase PF00009 GTP EFTU PF01399 PCI $c^{2}$ s. $^{2} \sin ^{2}$ PF00125 Histone

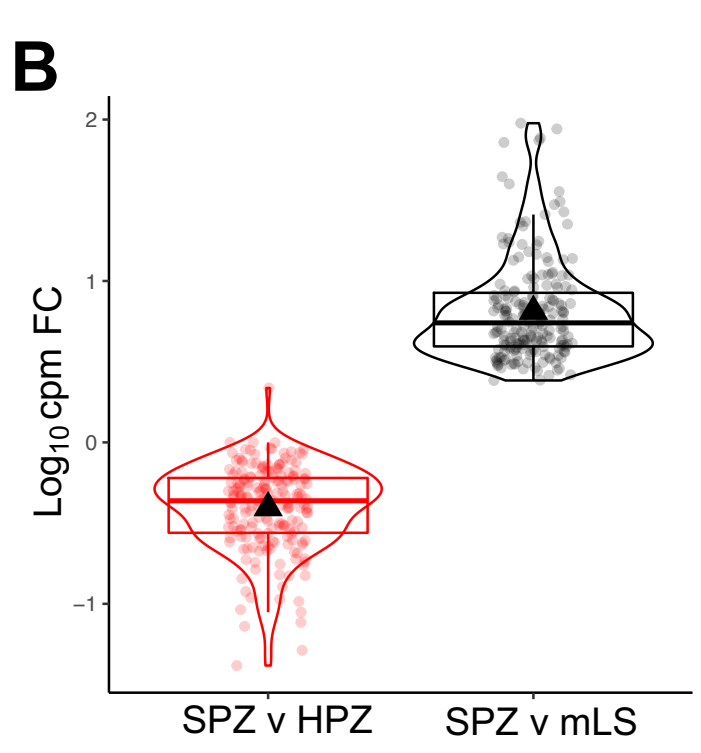

\section{SPZ enriched mLS enriched} \begin{tabular}{|lllllll|}
\hline & 1 & 1 & 1 & 1 & 1 \\
5 & 6 & 7 & 8 & 9 & 10
\end{tabular} $\begin{array}{ccc}6 & 7 & 8 \\ \log 10 & \mathrm{CPM}\end{array}$

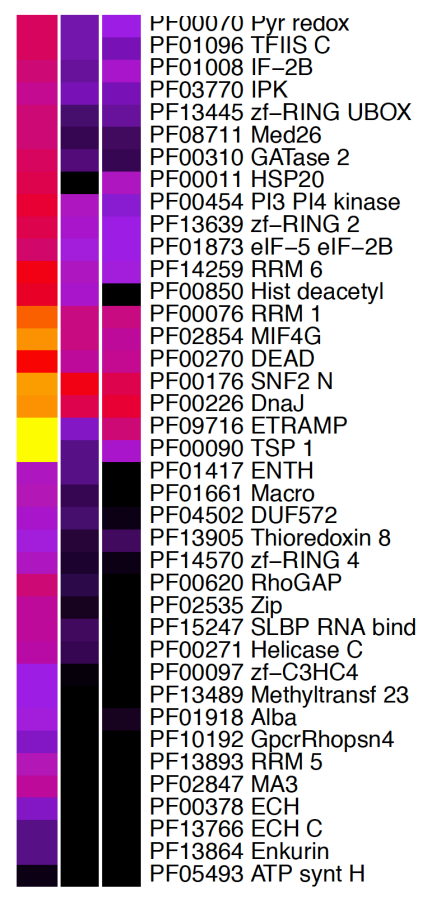
की ते $^{2} \hat{R}^{2}$

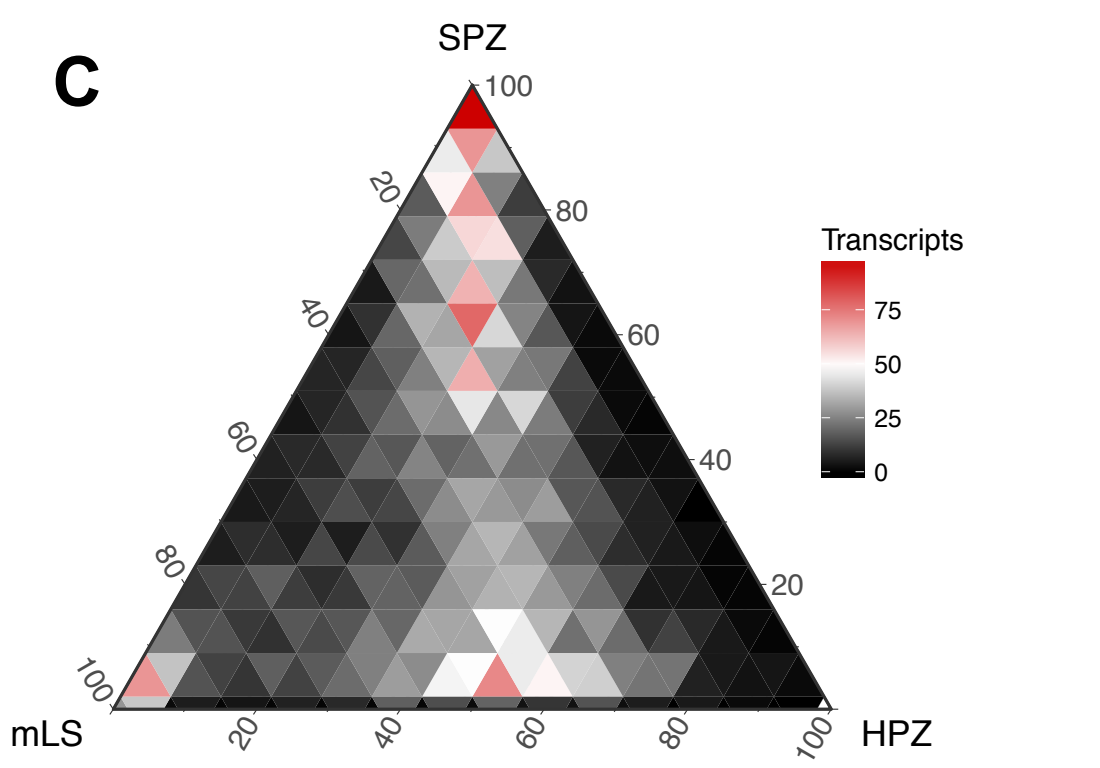




\section{DIFFERENTIATION}

- Translational repressors

e.g., Puf2, Alba2/4, HOMU, Yippee, Zipco

- Invasion

e.g., Celtos, Gest

- Liver development e.g., LISP1, LISP2, ETRAMPS, TRAP

- Transcription factors e.g., AP2-SP2, AP2-L, AP2-Q?

\section{SUPPRESSION}

- Metabolic/replication suppressors e.g., sucB, HAP2, MAK16

- Histone arginine methylation e.g., CARM1, EEML2

- Protein translation e.g., elF-3H, Puf1

\section{ACTIVATION}

- Merozoite development e.g., MSP1, MSP3, MSP9

- Rhoptry function e.g., RAP1, RNP2, RNP3

- Reticuocytye binding e.g., RBP2a, RBP2b, RBP2C

- Exported Proteins e.g., PHISTs

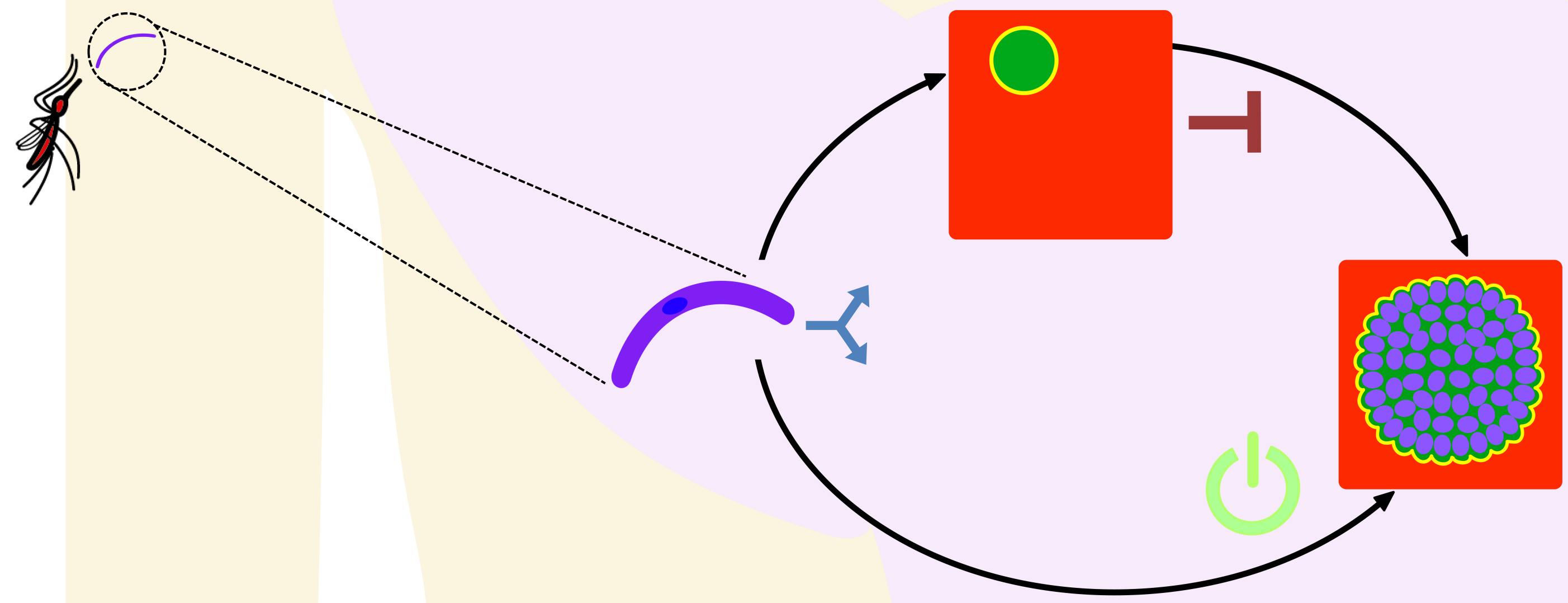

